\title{
Concentration Phenomena of Two-Vortex Solutions in a Chern-Simons Model
}

\author{
CHIUN-CHUAN CHEN - CHANG-SHOU LIN - GUOFANG WANG
}

\begin{abstract}
By considering an Abelian Chern-Simons model, we are led to study the existence of solutions of the Liouville equation with singularities on a flat torus. A non-existence and degree counting for solutions are obtained. The former result has an application in the Chern-Simons model.
\end{abstract}

Mathematics Subject Classification (2000): 35J60 (primary); $58 \mathrm{E} 11$ (secondary).

\section{1. - Introduction}

The Abelian Chern-Simons Higgs model introduced by Hong-Kim-Pac [18] and Jackiw-Weinberg [19] has the Lagrangian density

$$
\mathcal{L}(\phi, A)=D_{\alpha} \phi \overline{D_{\alpha} \phi}+\frac{1}{4} \kappa \varepsilon^{\alpha \beta \gamma} F_{\alpha \beta} A_{\gamma}-V(|\phi|)
$$

for a complex scalar (Higgs) field $\phi$ coupled with a Chern-Simons gauge field $A$ on $2+1$ dimensional Minkowski space $\mathbb{R}^{1,2}$. Here $F_{\alpha \beta}$ are the component of the curvature $F$ of $A, D_{\alpha} \phi=\partial_{\alpha} \phi-i A_{\alpha} \phi$ and $\kappa$ is a coupling parameter. The Euler-Lagrange equation for the Lagrangian action density $\mathcal{L}$ is the following system

$$
\begin{aligned}
\frac{1}{2} \kappa \varepsilon^{\alpha \beta \gamma} F_{\alpha \beta} & =j^{\gamma}=i\left(\phi \overline{D^{\gamma} \phi}-\bar{\phi} D^{\gamma} \phi\right), \\
D_{\alpha} D^{\alpha} \phi & =-\frac{\partial V(\phi)}{\partial \phi},
\end{aligned}
$$

where $j^{\gamma}$ is the conserved matter current density. We are interested in timeindependent vortex solutions to these field equations (1.2). For such static configuration, the energy becomes

$$
E(\phi, A)=\int\left(\left|D_{A} \phi\right|^{2}-A_{0}^{2}|\phi|^{2}-\kappa A_{0} F^{12}+V(|\phi|)\right) d^{2} x .
$$

Pervenuto alla Redazione il 23 settembre 2003 e in forma definitiva il 29 marzo 2004. 
Varying it w.r.t $A_{0}$ yields

$$
A_{0}=-\frac{\kappa}{2} \frac{F^{12}}{|\phi|^{2}},
$$

(the Gauss law) and $E$ becomes

$$
E(\phi, A)=\int\left(\left|D_{A} \phi\right|^{2}+\frac{\kappa^{2}}{4} \frac{|F|^{2}}{|\phi|^{2}}+V(|\phi|)\right) d^{2} x .
$$

With the choice $V(\phi \mid)=\frac{1}{\kappa^{2}}|\phi|^{2}\left(1-|\phi|^{2}\right)^{2}$, (1.4) may be rewritten as

$$
\begin{aligned}
E(\phi, A)= & \int\left(\left|\left(D_{1} \pm D_{2}\right) \phi\right|^{2}+\left(\frac{\kappa}{|\phi|} F_{12} \mp \frac{2}{\kappa}|\phi|\left(|\phi|^{2}-1\right)\right)^{2}\right. \\
& \left. \pm F_{12}+\operatorname{Im}\left\{\partial_{j} \varepsilon_{j k} \bar{\phi} D_{k} \phi\right\}\right) d^{2} x
\end{aligned}
$$

For this model, see [18] and [19] or [17].

Note that $E$ is gauge invariant in the sense that its value is unaffected under changing $(\phi, A)$ to $\left(e^{i \vartheta} \phi, A+\partial \vartheta\right)$ with real valued $\vartheta$. Following CaffarelliYang [5], we now want to study solutions that are periodic w.r.t. some lattice on $\mathbb{R}^{2}$. Such solutions can be interpreted as solutions on a fundamental domain

$$
\Omega=\left\{\tau \omega_{1}+\tau_{2} \omega_{2}, 0<\tau_{1}, \tau_{2}<1\right\}
$$

for the lattice, where $\omega_{1}=a, \omega_{2}=i b$, and $a, b \in \mathbb{R}$, satisfying the so-called ' $\mathrm{t}$ Hooft boundary conditions. See [5], [15] and [24]. Then it leads us to consider the following energy functional on a flat torus

$$
E(\phi, A)=\int d^{2} x\left\{\left|\left(D_{1}+i D_{2}\right) \phi\right|^{2}+\left(\frac{\kappa}{2} \frac{F_{12}}{|\phi|}-\frac{1}{\kappa}|\phi|\left(|\phi|^{2}-1\right)\right)^{2}\right\}+2 \pi N
$$

The absolute minima of $E$ therefore satisfy the Bogomolny type self-dual system

$$
\left\{\begin{array}{l}
D_{1} \phi+i D_{2} \phi=0 \\
\kappa^{2} F_{12}=2|\phi|^{2}\left(1-|\phi|^{2}\right) .
\end{array}\right.
$$

subject to the 't Hooft boundary condition. Here $\phi$ ( $A$ resp.) can be interpreted as a section (a connection resp.) of a line bundle over a compact flat torus of degree $N . N$ is the vortex number and the zero points of $\phi$ is referred as to vortex points. There are two types of solutions of (1.7) as $\kappa \rightarrow 0$ : (a) $|\phi|^{2} \rightarrow 1$ and (b) $|\phi|^{2} \rightarrow 0$ in some suitable topology. For the first type solutions, we refer to [5]. Recently, there has been some interest in finding the second type solutions with given vortex points. See [16] and [24] for $N=1,2$ and [16], 
[23 for general situations. In this papaer we are interested in the second type solutions, which resemble "non-topological" solutions in $\mathbb{R}^{2}$.

Following an approach introduced by Taubes in [28], one can rewrite the self-dual system (1.7) as a scalar equation. By introducing

$$
\begin{aligned}
\phi(x) & =\exp \left[\frac{v(x)}{2}+i \sum_{j=1}^{N} \arg \left(x-p_{j}\right)\right], \\
A_{1}+i A_{2} & =-2 i \bar{\partial} \log \phi,
\end{aligned}
$$

we have

$$
\Delta v+\frac{4}{\kappa^{2}} e^{v}\left(e^{v}-1\right)-4 \pi \sum_{j=1}^{N} \delta_{p_{j}}=0 .
$$

where $\left\{p_{1}, \ldots, p_{N}\right\}$ is the set of vortex points of $\phi, \delta_{p_{j}}$ is the Dirac measure with singularity at $p_{j}$ and $v(x)$ is a real-valued function.

We are interested in the asymptotic behaviors of the second type of solutions of (1.8), i.e, $v(x) \rightarrow-\infty$, as $\kappa \rightarrow 0$. Consider the simplest case $N=2$ and $p=p_{1}=p_{2}$. Set $G(x, p)$ to be the Green function defined by

$$
\left\{\begin{array}{l}
-\Delta G(x, p)=\delta_{p}-1, \\
\int_{\Omega} G(x, p) d x=0,
\end{array}\right.
$$

where for simplicity the volume of $\Omega$ is assumed to be 1 . By letting

$$
u(x)=v(x)+8 \pi G(x, p),
$$

equation (1.8) can be rewritten as

$$
\Delta u+\frac{4}{\kappa^{2}} h(x) e^{u}\left(h(x) e^{u(x)}-1\right)=8 \pi \quad \text { on } \Omega,
$$

where

$$
h(x)=\exp \left(-8 \pi G\left(x, p_{j}\right)\right) .
$$

Note that $u(x)$ is a smooth solution of (1.11) and $h(x)$ vanishes at $p$. Interestingly, in [24], Nolasco and Tarantello found that the asymptotic behaviors of solutions, as $\kappa \rightarrow 0$, might be related to the existence of minimizers of the following nonlinear functional $J$

$$
J(w)=\frac{1}{2} \int_{\Omega}|\nabla w|^{2}-8 \pi \log \left(\int_{\Omega} h e^{w}\right),
$$

where $w \in \stackrel{\circ}{H^{1}}=\left\{w \in H^{1} \mid \int w=0\right\}$ and $H^{1}$ is the Sobolev space of functions with $L^{2}$-integrable first derivatives. More precisely, they constructed 
a sequence of solution $u_{\kappa}$ of (1.11) such that $u_{\kappa}=w_{\kappa}+c_{\kappa}$ with $\int_{\Omega} w_{\kappa}=0$ and $u_{\kappa}+G(x, p) \leq 0$ on $\Omega$, satisfying

(a) $J\left(w_{\kappa}\right) \rightarrow \inf _{H^{1}}^{\circ} J$,

(b) $J$ attains its infimum on $\stackrel{\circ}{H}^{1}$ if and only if $w_{\kappa}$ is uniformly bounded on $\stackrel{\circ}{H}^{1}$

(c) Either (1.12) attains its infimum on $\stackrel{\circ}{H}^{1}$, then $w_{\kappa} \rightarrow w$ in $C^{l}(\Omega) \forall l \geq 0$, with $w \in \stackrel{\circ}{H}^{1}$ is a minimizer of $J$; or, the infimum of $J$ can not be attained on $\stackrel{\circ}{H^{1}}$ and

$$
\frac{h(x) e^{w_{\kappa}(x)}}{\int_{\Omega} h(x) e^{w_{\kappa}(x)}} \rightarrow \delta_{q} \text { in the sense of measure }
$$

for some $q \in \Omega$ and $q \neq p$.

In the terminology of gauge field, the second alternative in (c) above means that there exists a sequence of solutions $\phi_{\kappa}$ and $A_{\kappa}$ such that the flux density $F_{12}\left(\phi_{\kappa}, A_{\kappa}\right) \rightarrow 4 \pi \delta_{q}$ in the sense of measure. Obviously, the second alternative in (c) indicates that the concentration phenomenon can ocuur in the ChernSimons-Higg model. We remark that for non-topological solutions in $\mathbb{R}^{2}$, a similar phenomenon also occurs. For more precise statements of the above results, see [7]. Therefore, it is interesting to know if $J$ attains its infimum or not. One of our purposes of this paper is to give an answer to this question.

THEOREM 1.1. The nonlinear function $J$ cannot attain its infimum on $\stackrel{\circ}{H}^{1}$.

We note that the non-existence of minimizers of $J$ is indeed involved with a blow-up problem. By the Moser-Trudinger inequality, the nonlinear functional,

$$
J_{\rho}(w)=\frac{1}{2} \int_{\Omega}|\nabla w|^{2}-\rho \log \left(\int_{\Omega} h(x) e^{w}\right)
$$

where $h(x) \geq 0$ is a $C^{1}$ function, is bounded from below as long as $\rho \leq 8 \pi$. One way to look for a minimizer for $J$ is to ask whether a sequence of minimizers $v_{j}$ of $J_{8 \pi-\varepsilon_{j}}$ converges or not as $\varepsilon_{j} \downarrow 0$. It was proved that if $v_{j}$ blows up at some point $q$, then $h$ must satisfy $-\Delta \log h(q) \geq 8 \pi$, that is, if $h$ satisfies

$$
\Delta \log h(q)+8 \pi>0
$$

then the sequence of minimizers has a convergent subsequence. Now take our example $h(x)=\exp -(4 \pi N G(x, p))$. It is easy to see that the blow-up point $q \neq p$ and

$$
\Delta \log h(q)+8 \pi=4 \pi(2-N) .
$$

Hence if $N<2$, then by (1.14), the infimum of $J_{8 \pi}$ can be attained. On the other hand, for $N \geq 2$ (1.14) fails to guarantee the compactness of the 
sequence $v_{j}$. In general, we may consider $v_{j}$ to be a critical point of $J_{\rho_{j}}$ with $\lim _{j \rightarrow+\infty} \rho_{j}=8 \pi$, that is, $v_{j}$ satisfies

$$
\Delta v+\rho\left(\frac{h(x) e^{v}}{\int h e^{v}}-1\right)=0 \text { in } M
$$

with $\rho=\rho_{j}$, where $M$ is a compact Riemann surface and $\operatorname{vol}(M)=1$. Let

$$
\lambda_{j}:=\max _{M} v_{j}-\log \left(\int h e^{v_{j}}\right)=v_{j}\left(q_{j}\right)-\log \left(\int h e^{v_{j}}\right) .
$$

Suppose $\lambda_{j} \rightarrow+\infty$. In [11], the first two authors proved that

$$
\rho_{j}-8 \pi=\frac{2}{h\left(q_{j}\right)}\left(\Delta \log h\left(q_{j}\right)+8 \pi-2 K\left(q_{j}\right)\right) \lambda_{j} e^{-\lambda_{j}}+O\left(e^{-\lambda_{j}}\right)
$$

holds, where $K$ is the Gaussian curvature. When $M$ is a flat torus and $h(x)=$ $\exp -(4 \pi N G(x, p))$, we have

$$
\Delta \log h\left(q_{j}\right)+8 \pi-2 K\left(q_{j}\right)=4 \pi(2-N) .
$$

Thus, if $N \neq 2$, we have the important information about the sign of $\rho_{j}-8 \pi$. This sign condition is important when we come to the question of compactness of solutions of (1.15) with $\rho=8 \pi$, and the computation of topological degree of (1.15) for $\rho=8 \pi$ or $\rho>8 \pi$. For the precise definition of topological degree of (1.15) and related results, see [12] and references therein. Here for convenience of the reader, we sketch it as follows. It is well-known now that the set of solutions of equation (1.15) is compactness if $\rho \neq 8 \pi \mathrm{m}$. Hence, in this case there is a constant $C>0$ such that $\|u\|_{H^{1}}<C$ for any solution $u$ of (1.15). Set

$$
T(\rho)=\rho \Delta^{-1}\left(\frac{h(x) e^{v}}{\int h e^{v}}-1\right)
$$

which acts on $\stackrel{\circ}{H}^{1}$. The topological degree $d_{\rho}$ of (1.15) is defined to be the Leray-Schauder degree

$$
d_{\rho}:=\operatorname{deg}\left(I d+T(\rho), B_{R}, 0\right),
$$

for a large number $R$, where $B_{R}=\left\{v \in \stackrel{\circ}{H}^{1} \mid\|v\|_{H^{1}} \leq R\right\}$. For $\rho=8 \pi m$, if the solution space is compact, one can also define the topological degree.

Case $N=2$ is again the most delicate situation because (1.16) could not provide any useful information. Thus, we need to refine our previous estimate in [11] to prove the following compactness result. 
THEOREM 1.2. Suppose $h(x)=\exp (-8 G(x, p))$ and $M=\Omega$. Then there exists a constant $C$ such that

$$
|v(x)| \leq C \text { for } x \in \Omega
$$

holds for any solution $v$ of (1.15) with $\rho=8 \pi$. Furthermore, the topological degree for (1.15) with $\rho=8 \pi$ vanishes.

Theorem 1.1 and Theorem 1.2 strongly suggest that the nonlinear functional $J$ has no critical points. But so far, it remains an open problem.

Equation (1.15) arises naturally in many models in mathematical physics and differential geometry. When $M$ is the sphere and $\rho=8 \pi$, it is the Nirenberg problem. In $\mathbb{R}^{2}$, it also appears in the mean field limit of Euler flows, see [6], [8] and [22]. Therefore, it is important to study equation (1.15) for a general parameter $\rho \in \mathbb{R}$. In [12], an existence theorem has been studied when $h(x)$ is strictly positive. In fact, the topological degree for (1.15) has been calculated when $h(x)>0$. Since $h(x)$ vanishes somewhere in many applications, we are lead naturally to consider that at each $p$ with $h(p)=0, h(x)$ satisfies

$$
h(x)=|x-p|^{2 \alpha} h_{1}(x) \text { in a neighborhood of } p,
$$

where $\alpha>0$ and $h_{1}(x)$ is $C^{1}$ positive function. The number $\alpha$ is called the vanishing order of $h$ at $p$. In this paper, we consider the case $\rho \in(-\infty, 16 \pi)$.

THEOREM 1.3. Let $h(x)$ be a continuous non-negative function on $M$ and (1.17) holds at any $p$ where $h(p)=0$. Suppose the vanishing order at any $p$ with $h(p)=0$ is no less than 1 . Then for any $\rho \in(-\infty, 8 \pi) \cup(8 \pi, 16 \pi)$, there exists a constant $C_{\rho}>0$ such that

$$
|v(x)| \leq C_{\rho} \text { in } M
$$

holds for any solution $v$ of (1.15). Furthermore, the topological degree $d_{\rho}$ of (1.15) is given by

$$
d_{\rho}=\left\{\begin{array}{l}
1 \rho \in(-\infty, 8 \pi), \\
2 g-1+m \rho \in(8 \pi, 16 \pi),
\end{array}\right.
$$

where $m$ is the number of $\{x \in M \mid h(x)=0\}$ and $g$ is the genus of $M$.

We note that $d_{\rho}$ vanishes for $\rho \in(8 \pi, 16 \pi)$ only when $g=0$ and $N=1$. Therefore, solutions of (1.15) exist for $8 \pi<\rho<16 \pi$ when either $M$ is sphere and $m \geq 2$ or the genus of $M \geq 1$. The existence theorem for the latter has been proved in [2].

In this article, we are also interested in the following related problem

$$
\left\{\begin{array}{l}
\Delta v+\rho \frac{e^{v}}{\int_{\Omega} e^{v}}=4 \pi \sum_{j=1}^{m} n_{j} \delta_{p_{j}} \text { in } \Omega, \\
v=0 \text { on } \partial \Omega .
\end{array}\right.
$$

where $n_{j}$ is a positive integer and $\Omega$ is a bounded smooth domain in $\mathbb{R}^{2}$. It is known that for the case $m=0$, there exists no solution of (1.19) when $\Omega$ is a ball and $\rho \geq 8 \pi$. In fact, it is proved the topological degree of (1.19) vanishes when $\rho \in(8 \pi, \infty) \backslash\{8 k \pi \mid k=1,2, \ldots\}$ and $\Omega$ is simply connected, if $m=0$. In contrast, we have the following result when $m \geq 1$. 
THEOREM 1.4. Suppose that $\Omega$ is a bounded smooth domain in $\mathbb{R}^{2}$ and $m \geq 1$. Then equation (1.19) always possesses a solution for $\rho \in(8 \pi, 16 \pi)$.

The paper is organized as follows. In section 2, the non-existence of minimizers of $J$ is proved. In our proof, we will employ the geometric properties of the Liouville equation, especially the theory of elliptic functions. The symmetry property of minimizers also plays a crucial role in our proof. Without a surprise, we use a variant of the method of moving planes to establish the symmetry of solutions. Here we remark that the minimality is used in order to start the process, which is different from the situation for the Dirichlet problem in $\mathbb{R}^{n}$. A refined estimate of (1.16) and also the part of, the uniform boundedness in Theorem 1.2 are proved in Section 3, where again, the Weierstrass $\wp$ function plays the essential role. In Section 4, we are going to prove the degree counting formulas for Theorem 1.2, Theorem 1.3 and Theorem 1.4, where the results in [11] and [12] are used.

ACKNOWLEDGEMENTS. Part of this paper was carried out while the third author was visiting National Center of Theoretical Sciences in Taiwan and Mathematics Department of Chung Cheng University. He would like to thank them for warm hospitality. We also would like to thank the referee for his /or her careful reading.

\section{2. - Non-existence of minimizers}

In this section, we are going to prove Theorem 1.1, that is, the non-existence of minimizers of the functional $J$ of (1.12). We first have to prove some symmetry property of the Green function $G(x, q)$. Recall that the fundamental domain $\Omega$ of the torus is $\left[-\frac{a}{2}, \frac{a}{2}\right] \times\left[-\frac{b}{2}, \frac{b}{2}\right]$ and $G(x, q)$ denotes the Green function of $-\Delta$. Let $\omega_{1}=a$ and $\omega_{2}=i b$ with $i=\sqrt{-1}$. In the following, we also use a complex number $z=x_{1}+i x_{2}$ to denote a point $\left(x_{1}, x_{2}\right)$ in $\mathbb{R}^{2}$. When there is no ambiguity, we use $G(x)$ to denote the Green function with its pole at 0 .

$$
\text { LEMMA 2.1. Let } z=x_{1}+i x_{2} \text {. }
$$

(a) If $x_{2}$ is fixed, then $G(z)$ is strictly increasing in $x_{1}$ for $-\frac{1}{2} a<x_{1}<0$ and strictly decreasing in $x_{1}$ for $0<x_{1}<\frac{1}{2} a$. If $x_{1}$ is fixed, then $G(z)$ is strictly increasing in $x_{2}$ for $-\frac{1}{2} b<x_{2}<0$ and strictly decreasing in $x_{2}$ for $0<x_{2}<\frac{1}{2} b$. Moreover, $G(z)$ is symmetric with respect to the axis $x_{1}=0$ and the axis $x_{2}=0$.

(b) $G(z)$ has only three critical points $\frac{1}{2}\left(\omega_{1}+\omega_{2}\right), \frac{1}{2} \omega_{1}$ and $\frac{1}{2} \omega_{2}$. All of them are 
non-degenerate. Moreover, $\frac{1}{2}\left(\omega_{1}+\omega_{2}\right)$ is a minimum point, and $\frac{1}{2} \omega_{1}$ and $\frac{1}{2} \omega_{2}$ are saddle points. and

PROOF. Let $z^{*}$ be the reflection point of $z$ with respect to the axis $x_{1}=0$

$$
G^{*}(z)=G\left(z^{*}\right) .
$$

Then $G^{*}$ is a Green function of $-\Delta$ also. By the uniqueness of Green's function, we have $G^{*}=G$. Hence $G$ is symmetric with respect to the axis $x_{1}=0$. The same argument implies that $G$ is symmetric with respect to the line $x_{1}=m \frac{a}{2}$, for any integer $m$.

Consider $G_{x_{1}}=\frac{\partial G}{\partial x_{1}}$ in $\mathbb{R}^{2} . G_{x_{1}}$ is a harmonic function for $0 \leq x_{1} \leq \frac{a}{2}$ with singularities at $m \omega_{2}$, where $m$ is an integer. By the symmetry of $G$, we have $G_{x_{1}}=0$ on axes $x_{1}=0$ and $x_{1}=\frac{a}{2}$ except at the singularities.

We claim that

$$
G_{x_{1}}<0 \quad \text { for } 0<x_{1}<\frac{a}{2} \text {. }
$$

If this is not true, there exists a global maximum point $y=y_{1}+i y_{2}$ with $0<y_{1}<\frac{a}{2}$ such that $G_{x_{1}}(y)>0$. Since $\liminf _{x \rightarrow 0, x_{1} \geq 0} G_{x_{1}}(z) \leq 0$, this contradicts the maximum principle.

A similar argument can be applied to show that $G_{x_{2}}<0$ for $-\frac{b}{2}<x_{2}<0$. Therefore by the symmetry of $G$, in the fundamental domain the only critical points of $G$ are $\frac{\omega_{1}+\omega_{2}}{2}, \frac{\omega_{1}}{2}$ and $\frac{\omega_{2}}{2}$.

To show that the Hessian of $G$ at a critical point is not degenerate, we use the Hopf boundary point lemma. Consider $G_{x_{1}}$ in the domain $0<x_{1}<\frac{a}{2}$. Then $G_{x_{1}}\left(\frac{\omega_{1}+\omega_{2}}{2}\right), G_{x_{1}}\left(\frac{\omega_{2}}{2}\right), G_{x_{1}}\left(\frac{\omega_{1}}{2}\right)$ are local maxima at the boundary. By the Hopf boundary point lemma, $G_{x_{1} x_{1}}\left(\frac{\omega_{1}+\omega_{2}}{2}\right)>0, G_{x_{1} x_{1}}\left(\frac{\omega_{2}}{2}\right)>0$ and $G_{x_{1} x_{1}}\left(\frac{\omega_{1}}{2}\right)<0$. By a similar argument, we have $G_{x_{2} x_{2}}\left(\frac{\omega_{1}+\omega_{2}}{2}\right)>0, G_{x_{2} x_{2}}\left(\frac{\omega_{1}}{2}\right)>0$ and $G_{x_{2} x_{2}}\left(\frac{\omega_{2}}{2}\right)<0$. Since $G_{x_{1}}=0$ on $x_{1}=\frac{1}{2} \omega_{1}$, we have $G_{x_{1} x_{2}}\left(\frac{\omega_{1}+\omega_{2}}{2}\right)=0$ and $G_{x_{1} x_{2}}\left(\frac{\omega_{2}}{2}\right)=0$. Similarly, we have $G_{x_{1} x_{2}}\left(\frac{\omega_{1}}{2}\right)=0$. From these facts, we conclude that $G$ is non-degenerate at critical points and the proof is complete.

Rewrite the functional $J$ as

$$
J(v)=\frac{1}{2} \int_{\Omega}|\nabla v|^{2}+8 \pi \int_{\Omega} v-8 \pi \log \int_{\Omega} e^{-8 \pi G} e^{v}
$$

in $H^{1}(\Omega)$. It is clear that the Euler-Lagrange equation of $J$ is

$$
-\Delta v=8 \pi\left(\frac{e^{-8 \pi G} e^{v}}{\int_{\Omega} e^{-8 \pi G} e^{v}}-1\right) .
$$

By applying the symmetry property of the Green function, we obtain the following symmetry property of minimizers of $J$. 
Lemma 2.2. Suppose $v$ is a minimizer of $J$ in $\stackrel{\circ}{H}^{1}$. Then $v$ satisfies

$$
v\left(x_{1},-x_{2}\right)=v\left(x_{1}, x_{2}\right) \text { and } v\left(-x_{1}, x_{2}\right)=v\left(x_{1}, x_{2}\right) .
$$

PROOF. We will apply a variant of the well known method of moving planes to prove the desired results. First, we note that by using the comparison function $v+t \phi, \phi \in H^{1}$, the minimizer $v$ satisfies

$$
\int|\nabla \phi|^{2}-8 \pi \frac{\int e^{-8 \pi G} e^{v} \phi^{2} d x}{\int e^{-8 \pi G} e^{v} d x}+8 \pi\left(\frac{\int e^{-8 \pi G} e^{v} \phi d x}{\int e^{-8 \pi G} e^{v} d x}\right)^{2} \geq 0
$$

for any $\phi \in H^{1}$. By using (2.4), we could prove Lemma 2.1.

In the following, we only give a proof of $v(-x, y)=v(x, y)$, since the argument for the other identity is the same.

Let $\Omega_{ \pm}:=\left\{\left(x_{1}, x_{2}\right) \in\left[-\frac{\omega_{1}}{2}, \frac{\omega_{1}}{2}\right] \times\left[-\frac{\omega_{2}}{2}, \frac{\omega_{2}}{2}\right] \mid \pm x_{1}>0\right\}$. For $x \in \Omega_{+}$, let $x^{*}=-x$. We claim that one of the following alternatives holds: (a) $v(x)<v\left(x^{*}\right)$ for all $x \in \Omega_{+}$; (b) $v(x)>v\left(x^{*}\right)$ for all $x \in \Omega_{+}$, and (c) $v(x)=v\left(x^{*}\right)$ for all $x \in \Omega_{*}$.

Assume by contradiction that the claim is not true. Namely, the following two sets are both nonempty:

$$
D_{+}=\left\{x \in \Omega_{+} \mid v(x)>v\left(x^{*}\right)\right\} \quad \text { and } \quad D_{-}=\left\{x \in \Omega_{+} \mid v(x)<v\left(x^{*}\right)\right\} .
$$

Set $w(x)=v(x)-v\left(x^{*}\right)$. It is clear that $w$ satisfies

$$
\Delta w(x)+8 \pi e^{-8 \pi G(x)} c(x) w=8 \pi\left(e^{-8 \pi G\left(x^{*}\right)}-e^{-8 \pi G(x)}\right) e^{v\left(x^{*}\right)+c_{0}}=0,
$$

where $e^{c_{0}}=\left(\int_{\Omega} e^{-8 \pi G} e^{v}\right)^{-1}$ and $c(x)=\frac{e^{v(x)+c_{0}-e^{v\left(x^{*}\right)+c_{0}}}}{v(x)-v\left(x^{*}\right)}$. The last equality in (4.2) follows from the symmetry of $G$. Set $D_{-}^{*}=\left\{x^{*} \mid x \in D_{-}\right\} \subseteq \Omega_{-}$. We define a new function $\phi$ in $\Omega$ by

$$
\phi(x)= \begin{cases}w(x), & x \in D_{+}, \\ c w(x), & x \in D_{-}^{*} \\ 0, & \text { otherwise }\end{cases}
$$

where $c$ is a positive constant such that

$$
\int_{\Omega} e^{-8 \pi G} e^{v+c_{0}} \phi(x) d x=0 .
$$

Since $1-e^{-w}-w<0$ for all $w>0$, it is easy to check that $\phi$ satisfies

$$
\Delta \phi+8 \pi e^{-8 \pi G} e^{v+c_{0}} \phi \begin{cases}>0, & x \in D_{+} \\ <0, & x \in D_{-}^{*} \\ =0 & \text { otherwise. }\end{cases}
$$


For instance, for $x \in D_{+}$we have

$$
c(x)=e^{c_{0}}\left(\frac{e^{v(x)}-e^{v\left(x^{*}\right)}}{v(x)-v\left(x^{*}\right)}\right)=e^{v+c_{0}}(x) \frac{1-e^{-w}}{w}<e^{v(x)+c_{0}},
$$

which, by (2.5), implies that $\Delta \phi+8 \pi e^{-8 \pi G} e^{v+c_{0}} \phi>0$. By (2.8), we have

$$
-\int_{\Omega}|\nabla \phi|^{2}+8 \pi \int_{\Omega} e^{-8 \pi G} e^{v+c_{0}} \phi^{2}>0
$$

which yields a contradiction to (2.4). Therefore the claim is proved.

From the claim, to prove the Lemma we only need to exclude cases (a) and (b). Now assume case (b) happens, i.e.,

$$
v(x)>v\left(x^{*}\right) \text { for all } x \in \Omega_{+} .
$$

Let $\lambda \in\left(0, \frac{\omega_{1}}{2}\right)$ and for $x \in\left[\lambda, \lambda+\frac{\omega_{1}}{2}\right] \times\left[-\frac{\omega_{2}}{2}, \frac{\omega_{2}}{2}\right]$ set $x_{\lambda}^{*}=2 \lambda-x$. We consider a family of blocks $\left[-\frac{\omega_{1}}{2}+\lambda, \frac{\omega_{1}}{2}+\lambda\right] \times\left[-\frac{\omega_{2}}{2}, \frac{\omega_{2}}{2}\right]$. Let $\Omega_{\lambda}^{+}=\left[\lambda, \frac{\omega_{1}}{2}+\right.$ $\lambda] \times\left[-\frac{\omega_{2}}{2}, \frac{\omega_{2}}{2}\right]$ and $\Omega_{\lambda}^{-}=\left[-\frac{\omega_{1}}{2}+\lambda, \lambda\right] \times\left[-\frac{\omega_{2}}{2}, \frac{\omega_{2}}{2}\right]$. Set $v_{\lambda}(x)=v\left(x_{\lambda}^{*}\right)$ and define $w_{\lambda}=v(x)-v_{\lambda}(x)$ for $x \in \Omega_{\lambda}^{+}$. It is clear that $\omega_{\lambda}$ satisfies

$$
\Delta w_{\lambda}(x)+8 \pi e^{-8 \pi G(x)} c(x) w_{\lambda}=8 \pi\left(e^{-8 \pi G\left(x_{\lambda}^{*}\right)}-e^{-8 \pi G(x)}\right) e^{v_{\lambda}(x)}, \quad x \in \Omega_{\lambda}^{+},
$$

where $c(x)=\frac{e^{v(x)}-e^{v_{\lambda}(x)}}{v(x)-v_{\lambda}(x)}$. To apply the maximum principle, we need to check that the left hand side of (2.11) is non-positive. This follows from Lemma 2.1, since $|x|>\left|x_{\lambda}^{*}\right|$ implies $G(x) \leq G\left(x_{\lambda}^{*}\right)$. By the maximum principle together with the standard argument of the method of moving planes, it is easy to prove that for any $\lambda \in\left(0, \frac{\omega_{1}}{2}\right), w_{\lambda}(x)>0$ for all $x \in \Omega_{\lambda}^{+}$and $w_{\frac{\omega_{1}}{2}}(x) \geq 0$ for all $x \in \Omega_{\frac{\omega_{1}}{2}}^{+}$. That is,

$$
v(x) \geq v\left(\omega_{1}-x\right) \quad \text { for } x \in\left[\frac{\omega_{1}}{2}, \omega_{1}\right] \times\left[-\frac{\omega_{2}}{2}, \frac{\omega_{2}}{2}\right] .
$$

We show that this yields a contradiction. Let $x \in\left(0, \frac{\omega_{1}}{2}\right) \times\left[-\frac{\omega_{2}}{2}, \frac{\omega_{2}}{2}\right]$ and $y=\omega_{1}-x$. It is clear that $y \in\left[\frac{\omega_{1}}{2}, \omega_{1}\right] \times\left[-\frac{\omega_{2}}{2}, \frac{\omega_{2}}{2}\right]$. By $(2.10)$ and periodicity of $v,(2.12)$ yields

$$
v(y) \geq v(x)>v(-x)=v\left(\omega_{1}-x\right)=v(y),
$$

which is a contradiction. Similarly, we can exclude case (a) and finish the proof of $v\left(-x_{1}, x_{2}\right)=v\left(x_{1}, x_{2}\right)$. 
For the rest of the proof, we use the geometric property of the Liouville equation to get a contradiction. We first recall a classical result of Liouville: Let $D$ be a simply-connected domain in $\mathbb{R}^{2}$ and $u$ is a solution of $\Delta u+e^{u}=0$ in $D$, then there exists a function $f$ analytic in $D$ such that

$$
u(z)=\log \frac{4\left|f^{\prime}\right|^{2}}{\left(1+|f|^{2}\right)^{2}} \quad \text { for } \quad z \in D
$$

For a domain $D$ and a solution $u$ of $\Delta u+e^{u}=0$ in $D$, an analytic function $f$ is called a developing map for $u$ in $D$, if (2.13) holds.

A straightforward computation shows that if $f$ is a developing map of $u$ in $D$, then

$$
u_{z z}(z)-\frac{1}{2} u_{z}^{2}(z)=\frac{f^{\prime \prime \prime}(z)}{f^{\prime}(z)}-\frac{3}{2}\left(\frac{f^{\prime \prime}(z)}{f^{\prime}(z)}\right)^{2} \quad \text { for } \quad z \in D
$$

Classically, the right hand side of (2.14) is called the Schwarz derivative of $f$. By (2.14), we see that any two developing maps $f$ and $\tilde{f}$ of $u$ has the same Schwarz derivative. Thus, $f$ and $\tilde{f}$ satisfy

$$
\tilde{f}(z)=\frac{a f(z)+b}{c f(z)+d} \quad \text { for } \quad z \in D
$$

where $a d-b c=1$. By direct computations, (2.13) together with (2.15) yields

$$
\tilde{f}(z)=\frac{p f(z)-\bar{q}}{q f(z)+\bar{p}} \quad \text { for } \quad z \in D
$$

where $p, q \in \mathbb{C}$ satisfies

$$
|p|^{2}+|q|^{2}=1
$$

i.e., $\left(\begin{array}{cc}p & -\bar{q} \\ q & \bar{p}\end{array}\right) \in P S U(2)$. (For an element $S=\left(\begin{array}{cc}p & -\bar{q} \\ q & \bar{p}\end{array}\right) \in P S U(2)$, sometimes we denote $S f=\frac{p f-\bar{q}}{q f+\bar{p}}$.) Thus, we have proved the following result.

LEMMA 2.3. Suppose that both $f$ and $\tilde{f}$ are developing maps of $u$ in $D$, where u satisfies

$$
\Delta u+e^{u}=0 \text { in } D .
$$

Then there exist $p, q, \in \mathbb{C}$ such that (2.16) and (2.17) hold.

We collect two useful properties concerning the transformation (2.16) in the followings. 
LEMMA 2.4. (a) Any element in PSU (2) is conjugate to $\left(\begin{array}{cc}e^{i \theta} & 0 \\ 0 & e^{-i \theta}\end{array}\right)$ for some $\theta$. Namely, for any $S=\left(\begin{array}{cc}p & -\bar{q} \\ q & \bar{p}\end{array}\right)$ there exists $H=\left(\begin{array}{cc}\alpha & -\bar{\beta} \\ \beta & \bar{\alpha}\end{array}\right)$ with $|\alpha|^{2}+|\beta|^{2}=1$ such that

$$
H^{-1} S H=\left(\begin{array}{cc}
e^{i \theta} & 0 \\
0 & e^{-i \theta}
\end{array}\right)
$$

for some $\theta \in \mathbb{R}$.

(b) Let $S_{1}=\left(\begin{array}{cc}e^{i \theta} & 0 \\ 0 & e^{-i \theta}\end{array}\right)$ and $S_{2}=\left(\begin{array}{cc}p & -\bar{q} \\ q & \bar{p}\end{array}\right)$ satisfying

$$
S_{1} S_{2}= \pm S_{2} S_{1}
$$

Then one of the following three possibilities occurs:

(i) $p=0$ and $e^{i \theta}= \pm i$,

(ii) $q=0$,

(iii) $e^{i \theta}= \pm 1$.

Proof. Part (a): It is easy to see that the eigenvalues of $S$ are conjugate $e^{ \pm i \theta}$ for some $\theta$. Unless $S= \pm i d, e^{i \theta} \neq e^{-i \theta}$. So, there exists an eigenvector $(\alpha, \beta)$ with $|\alpha|^{2}+|\beta|^{2}=1$ such that

$$
p \alpha-\bar{q} \beta=e^{i \theta} \alpha \quad \text { and } \quad q \alpha+\bar{p} \beta=e^{i \theta} \beta .
$$

By using the complex conjugate, the other equation for part (a) can be obtained. Part (b):

$$
S_{2} S_{1}=\left(\begin{array}{cc}
p e^{i \theta} & -\bar{q} e^{-i \theta} \\
q e^{i \theta} & \bar{p} e^{-i \theta}
\end{array}\right) \quad \text { and } \quad S_{1} S_{2}=\left(\begin{array}{cc}
p e^{i \theta} & -\bar{q} e^{i \theta} \\
q e^{-i \theta} & \bar{p} e^{-i \theta}
\end{array}\right)
$$

If $S_{2} S_{1}=S_{1} S_{2}$, then $q e^{-i \theta}=q e^{i \theta}$. Hence either $q=0$ or $e^{i \theta}= \pm 1$. If $S_{2} S_{1}=-S_{1} S_{2}$, then $p=0$ and $q e^{i \theta}=-q e^{-i \theta}$. Since $q \neq 0$, we have $e^{i \theta}= \pm i$.

In the application, we let $u=v-8 \pi G+c_{0}+\log (8 \pi)$, where $v$ is a minimizer of $J$ and $e^{-c_{0}}=\int_{\Omega} h(x) e^{v}$. Then $u$ satisfies $\Delta u+e^{u}=8 \pi \delta_{0}$, where $\delta_{0}$ is the Dirac measure with singularity at the origin. Now we will prove the existence of a developing function for $u$.

LEMMA 2.5. Suppose $u$ is a solution of

$$
\Delta u+e^{u}=8 \pi \delta_{0} \text { in } \Omega
$$

where $\Omega$ is the flat torus with the cell $\left[-\frac{a}{2}, \frac{a}{2}\right] \times\left[-\frac{b}{2}, \frac{b}{2}\right]$ and $\delta_{0}$ is the Dirac measure with singularity at the origin. Then there exists a meromorphic function $f$ in $\mathbb{C}$ such that (2.13) holds for $z \in \mathbb{C} \backslash\left\{n \omega_{1}+m \omega_{2} \mid n, m \in I\right.$. Here $I$ is the set of integers. 
ProOF. Lemma 2.5 is a known result. Basically, it was proved in [4] and [13]. For the convenience of reference, we give a proof here. We consider a punctured disk $D^{*}$ around a singularity, say 0 . Let $\tilde{D}^{*}=\{z \in \mathbb{C} \mid \operatorname{Re}(z)<0\}$ be the universal cover of $D^{*}$ with the covering map $z \rightarrow e^{z}$. Consider $u$ in $D^{*}$ and lift it to $\tilde{D}^{*}$. Denoting the lifted function by $\tilde{u}$, we have $\tilde{u}(z+2 \pi i)=\tilde{u}(z)$. By the Liouville theorem, we have a locally univalent meromorphic function $f$ in $\tilde{D}^{*}$, as a developing map of $\tilde{u}$. Since $\tilde{u}(z+2 \pi i)=\tilde{u}(z)$, we have

$$
f(z+2 \pi i)=\frac{p f(z)-\bar{q}}{q f(z)+\bar{p}}
$$

for some $p, q \in \mathbb{C}$ with $|p|^{2}+|q|^{2}=1$. See Lemma 2.3. Since any element in $P S U(2)$ is conjugate to $\left(\begin{array}{cc}e^{\theta i} & 0 \\ 0 & e^{-\theta i}\end{array}\right)$, by changing the developing map from $f$ to $H f$ by an element $H$ in $P S U(2)$, we may assume that

$$
f(z+2 \pi i)=e^{2 \pi i \alpha} f(z),
$$

for some $\alpha \in[0,1)$. Let $\psi(z)=e^{-\alpha z} f(z)$. By (2.18), it is clear that $\psi$ is a well-defined meromorphic function on $D^{*}$ and

$$
u=\log \frac{4|z|^{2(\alpha-1)}\left|\alpha \psi+z \psi^{\prime}\right|^{2}}{\left(1+\left|z^{\alpha} \psi\right|^{2}\right)^{2}}
$$

in $D^{*}$. By Lemma 2.4 of [13], if the origin is an essential singularity of $\psi$, then $f$ takes all values of $\mathbb{C}$ infinitely many times except at most one value. Since $\int_{D^{*}} e^{u}<+\infty$, we know that $\psi$ at most has a finite pole at 0 . Hence, there is an integer $n$ such that $z^{-n} \psi(z)=\tilde{\psi}(z)$ is a non-vanishing holomorphic function in a small neighborhood of 0 . Hence we have

$$
u=\log \frac{4|z|^{2(\alpha+n-1)}\left|(\alpha+n) \tilde{\psi}+z \tilde{\psi}^{\prime}\right|^{2}}{\left(1+\left|z^{\alpha+n} \tilde{\psi}\right|^{2}\right)^{2}}
$$

Now we apply the discussion above to our solution $u$. Applying the Liouville Theorem to $u$, we have a locally univalent (possibly multi-valued) meromorphic function $f$ as a developing map of $u$. Since $u=4 \log |z|$ near 0 , from (2.20) we have $\alpha=0$. Hence $f$ is single-valued. By continuation, $f$ can be globally defined in $\mathbb{C}$. This finishes the proof of Lemma 2.5.

REMARK 2.6. Let $f$ be a developing map for $u$. Then by (2.13), it is easy to see that at any point $n \omega_{1}+m \omega_{2}, n, m \in I$, either $f$ might be regular and $f^{\prime}$ has a zero of multiplicity 2 , or $f$ might have a pole there of multiplicity 3 . In particular, $f^{\prime}(z)$ has no zeros for $z \in \mathbb{C} \backslash\left\{n \omega_{1}+m \omega_{2} \mid n, m \in I\right\}$.

Now we are in the position to complete the proof of Theorem 1.1. 
Proof of Theorem 1.1. In view of Lemma 2.3, the periodicity of $u$ implies that there are elements $S_{1}$ and $S_{2} \in P S U$ (2) such that

$$
f\left(\omega_{1}+z\right)=S_{1} f(z) \quad \text { and } \quad f\left(\omega_{2}+z\right)=S_{2} f(z) .
$$

Since $f$ is single-valued, it is easy to show that $S_{1}$ and $S_{2}$ commute in $P S U(2)$, i.e.,

$$
S_{1} S_{2}= \pm S_{2} S_{1}
$$

In fact, we have $f\left(\omega_{1}+\omega_{2}+z\right)=S_{1} S_{2} f(z)=S_{2} S_{1} f(z)$. One can also assume that $S_{1}=\left(\begin{array}{cc}e^{i \theta} & 0 \\ 0 & e^{-i \theta}\end{array}\right)$ for some $\theta \in \mathbb{R}$. The reason is following. First, by Lemma 2.4, there exists $a \in P S U(2)$ such that $a \cdot S_{1} \cdot a^{-1}=\left(\begin{array}{cc}e^{i \theta} & 0 \\ 0 & e^{-i \theta}\end{array}\right)$. Second, for any $a \in P S U(2)$, we already know that $f_{a}=a f$ is also a developing map. Instead of $f$ we consider $f_{a}$. For $f_{a}$, by (2.21) we have

$$
f_{a}\left(\omega_{1}+z\right)=a \cdot S_{1} \cdot a^{-1} f_{a}(z) \quad \text { and } \quad f_{a}\left(\omega_{2}+z\right)=a \cdot S_{2} \cdot a^{-1} f_{a}(z) .
$$

The corresponding $\tilde{S}_{1}=a \cdot S_{1} \cdot a^{-1}$ has the required form.

Now set $S_{2}=\left(\begin{array}{cc}p & -\bar{q} \\ q & \bar{p}\end{array}\right)$. In view of (2.22), Lemma 2.4 gives one of three possibilities:

(i) $p=0$ and $e^{i \theta}= \pm i$.

(ii) $q=0$.

(iii) $e^{i \theta}= \pm 1$.

We get a contradiction by excluding all three cases.

CASE (i). In this case we have

$$
f\left(z+\omega_{1}\right)=-f(z) \quad \text { and } \quad f\left(z+\omega_{2}\right)=-\frac{\bar{q}}{q f(z)} .
$$

Hence $f$ is an elliptic function, i.e.,

$$
f\left(z+2 \omega_{1}\right)=f(z) \text { and } \quad f\left(z+2 \omega_{2}\right)=f(z) .
$$

To get a contradiction, we count the number of poles and zeros. There are two possibilities: either $f$ has a pole at 0 or $f$ is regular at 0 .

For the former case, by Remark 2.6 and (2.24), $f^{\prime}(z)$ has zeros only at $\omega_{2}, \omega_{1}+\omega_{2}$ with the multiplicity 2 . On the other hand, $f^{\prime}$ has at least poles at 0 and $\omega_{1}$ of multiplicity 4 . Because $f^{\prime}$ is an elliptic function with periods $2 \omega_{1}$ nd $2 \omega_{2}$, it leads to a contradiction.

If $f$ is regular at 0 , we assume $f(0)=0$ first. Then $f^{\prime}(z)$ has at lest poles at $\omega_{2}, \omega_{2}+\omega_{1}$ of multiplicity 4 and $f^{\prime}(z)$ exactly has zeros at $0, \omega_{1}$ of multiplicity 2 . Thus, it yields a contradiction.

If $f(0) \neq 0$, then $f$ is regular at $\omega_{1}, \omega_{2}$ and $\omega_{1}+\omega_{2}$ and $f$ has only simple poles somewhere. Clearly, $f^{\prime}$ has exactly zeros of multiplicity 2 at 0 , 
$\omega_{1}, \omega_{2}, \omega_{1}+\omega_{2}$. Hence $f^{\prime}$ has exactly four poles of multiplicity 2 , due to the fact that $f$ is an elliptic function in $\left[0,2 \omega_{1}\right] \times\left[0,2 \omega_{2}\right]$. Therefore, $f$ has exactly four simple poles. From here, we deduce that the total multiplicity of the solution $z$ of $f(z)=f(0)$ is four. Since 0 is a solution of $f(z)=f(0)$ of multiplicity 3 , there is a unique $z_{0} \neq 0$ satisfying $f\left(z_{0}\right)=f(0)$ and $f^{\prime}\left(z_{0}\right) \neq 0$. To get a contradiction, we apply the symmetry property of $v$. Since $v(-x)=v(x)$, we have $u(-x)=u(x)$. Therefore $f(-z)$ is also a developing map for $u$, by (2.15),

$$
f(-z)=\frac{p f(z)-\bar{q}}{q f(z)+\bar{p}}
$$

for some $p, q \in \mathbb{C}$ with $|p|^{2}+|q|^{2}=1$. Hence

$$
f(0)=\frac{p f(0)-\bar{q}}{q f(0)+\bar{p}}
$$

Since $f\left(z_{0}\right)=f(0),(2.25)$ yields,

$$
\begin{aligned}
f\left(-z_{0}\right) & =\frac{p f\left(z_{0}\right)-\bar{q}}{q f\left(z_{0}\right)+\bar{p}} \\
& =\frac{p f(0)-\bar{q}}{q f(0)+\bar{p}} \\
& =f(0)
\end{aligned}
$$

Thus, $z_{0}=-z_{0} \bmod 2 \omega_{1}$ and $2 \omega_{2}$, which implies $z_{0}=\omega_{1}, \omega_{2}$ or $\omega_{1}+\omega_{2}$. But this yields a contradiction to $f^{\prime}\left(z_{0}\right) \neq 0$, because $f^{\prime}\left(\omega_{j}\right)=0$, for $j=1,2,3$, where $\omega_{3}=\omega_{1}+\omega_{2}$. This contradiction finishees the proof of case (i).

CASE (ii). In this case, we have

$$
f\left(z+\omega_{1}\right)=e^{2 i \theta_{1}} f(z) \text { and } f\left(z+\omega_{2}\right)=e^{2 i \theta_{2}} f(z) .
$$

As before, either $f$ has a pole at 0 or $f(z)$ is regular at 0 . We consider the first situation first. Since $\left|f^{\prime}\right|$ has no zeros in $\left[0, \omega_{1}\right] \times\left[0, \omega_{2}\right]$ and is periodic with respect to $\omega_{1}$ and $\omega_{2}$, we have $\left|f^{\prime}(z)\right| \geq C>0$ for $z \in C$, for some positive constant $C$. Hence $f^{\prime}$ is a constant, which is also impossible.

If $f$ is regular at 0 , and $f(0)=0$, we can replace $f$ by $\frac{1}{f}$ and use the same argument as above to yield a contradiction.

Now it remains tp discuss the most delicate situation, when $f$ is regular at 0 and $f(0) \neq 0$. By $(2.28), \frac{f(z)}{f^{\prime}(z)}$ is an elliptic function on the torus $\left[0, \omega_{1}\right] \times$ $\left[0, \omega_{2}\right]$. Since 0 is the only pole of $\frac{f(z)}{f^{\prime}(z)}$ with multiplicity $2, \frac{f(z)}{f^{\prime}(z)}=A_{1} \wp(z)+A_{2}$ for some $A_{1}, A_{2} \in \mathbb{C}$ where $\wp(z)$ is the Weierstrass elliptic function. For a discussion on elliptic functions, see [1]. Thus, $\frac{f(z)}{f^{\prime}(z)}$ has two zeros only. We 
note that the zero of $\frac{f(z)}{f^{\prime}(z)}$ comes from either the zeros of $f$ or the poles of $f$. Let $z_{0}$ be zero of $\frac{f(z)}{f^{\prime}(z)}$. Clearly, $z_{0}$ must be a simple zero of $\frac{f}{f^{\prime}}$. Thus, $z_{0} \neq \frac{\omega_{1}}{2}, \frac{\omega_{2}}{2}, \frac{\omega_{1}+\omega_{2}}{2}$.

Since $\wp(-z)=\wp(z)$ and $\wp(\bar{z})=\wp(z), \frac{f(z)}{f^{\prime}(z)}$ satisfies

$$
\frac{f(-z)}{f^{\prime}(-z)}=\frac{f(z)}{f^{\prime}(z)}
$$

and

$$
\bar{A}_{3} \frac{\overline{f(\bar{z})}}{\bar{f}^{\prime}(\bar{z})}=A_{3} \frac{f(z)}{f^{\prime}(z)}+B
$$

where $\left|A_{3}\right|=1$ and $A_{3}, B \in \mathbb{C}$. From (2.29), we know that if $z_{0}$ is a zero of $\frac{f}{f^{\prime}}$, then $-z_{0}$ is another zero of $\frac{f}{f^{\prime}}$. So $z_{0}+n \omega_{1}+m \omega_{2}$ and $-z_{0}+n \omega_{1}+m \omega_{2}$ are all possible zeros and poles of $f$, where $n, m \in I$.

Since $f(-z)$ is a covering map for $u$, we have

$$
f(-z)=\frac{p_{1} f(z)-\bar{q}_{1}}{q_{1} f(z)+\bar{p}_{1}}
$$

with $\left|p_{1}\right|^{2}+\left|q_{1}\right|^{2}=1$. By direct computations, (2.31) yields

$$
\frac{f(z)}{f^{\prime}(z)}=\frac{f(-z)}{f^{\prime}(-z)}=\frac{-\left(q_{1} f(z)+\bar{p}_{1}\right)\left(p_{1} f(z)-\bar{q}_{1}\right)}{f^{\prime}(z)}
$$

Thus, we have

$$
p_{1} q_{1}=0 \quad \text { and } \quad 1=\left|q_{1}\right|^{2}-\left|p_{1}\right|^{2} .
$$

Therefore,

$$
p_{1}=0 \text { and }\left|q_{1}\right|=1
$$

and (2.31) is reduced to

$$
f(-z)=\frac{e^{2 i \theta_{3}}}{f(z)}
$$

for some $\theta_{3} \in[0, \pi)$. For simplicity, we may assume $f(0)=1$ after multiplying $f$ by a complex unit $e^{i \alpha}$. Then the identity above becomes

$$
f(-z)=\frac{1}{f(z)}
$$

Since $v\left(-x_{1},-x_{2}\right)=v\left(x_{1}, x_{2}\right), \overline{f(\bar{z})}$ is also a developing map for $u$. Thus,

$$
\overline{f(\bar{z})}=\frac{p_{2} f(z)-\bar{q}_{2}}{q_{2} f(z)+\bar{p}_{2}} .
$$


By (2.30), we have

$$
\begin{aligned}
A_{3} \frac{f(z)}{f^{\prime}(z)}+B & =\bar{A}_{3} \frac{\overline{f(\bar{z})}}{\overline{f^{\prime}(\bar{z})}} \\
& =\bar{A}_{3} \frac{\left(p_{2} f(z)-\bar{q}_{2}\right)\left(q_{2} f(z)+\bar{p}_{2}\right)}{f^{\prime}(z)}
\end{aligned}
$$

and

$$
B f^{\prime}(z)=\bar{A}_{3}\left(p_{2} f(z)-\bar{q}_{2}\right)\left(q_{2} f(z)+\bar{p}_{2}\right)-A_{3} f(z) .
$$

We claim $B=0$, because if $B \neq 0$, then $f(z)$ can directly be solved from (2.34) and the explicit expression of $f$ clearly yields a contradiction to (2.28). Since the argument is elementary, we only give a sketch of the proof for the case when the polynomial $\bar{A}_{3}\left(p_{2} \omega-\bar{q}_{2}\right)\left(q_{2} \omega+\bar{p}_{2}\right)-A_{3} \omega$ of the right hand of (2.34) is of order 2 and has two different roots. For other cases, the proof is simpler and we omit it here. For our situations, $f(z)$ can be solved by

$$
\log \frac{f(z)-a_{1}}{f(z)-b_{1}}=B_{1} z+B_{2},
$$

where $a_{1} \neq b_{1}$ and $B_{1} \neq 0$ because $B \neq 0$. Here $a_{1}, b_{1}$ are two roots of $\bar{A}_{3}\left(p_{2} \omega-\bar{q}_{2}\right)\left(q_{2} \omega+\bar{p}_{2}\right)-A_{3} \omega=0$. Thus $a_{1} \neq 0, b_{1} \neq 0$ because we assume $p_{2} q_{2} \neq 0$. Now by solving (2.35), we have

$$
f(z)=\frac{b_{1} A_{4} e^{B_{1} z}-a_{1}}{A_{4} e^{B_{1} z}-1}
$$

where $A_{4}=e^{B_{2}} \neq 0$. By using (2.28), we obtain

$$
e^{B_{1} \omega_{1}}=1 \text { and } e^{B_{1} \omega_{2}}=1,
$$

which is impossible.

Thus, we have $B=0$ and then

$$
p_{2} q_{2}=0 \quad \text { and } \quad \bar{A}_{3}\left(\left|p_{2}\right|^{2}-\left|q_{2}\right|^{2}\right)=A_{3} .
$$

Hence,

$$
\begin{aligned}
& \text { either } p_{2}=0,\left|q_{2}\right|=1 \quad \text { and } A_{3}= \pm i \\
& \text { or } \quad q_{2}=0,\left|p_{2}\right|=1 \quad \text { and } A_{3}= \pm 1 .
\end{aligned}
$$

The first case implies

$$
\overline{f(\bar{z})}=\frac{e^{2 i \theta_{4}}}{f(z)}
$$


By putting $z=0$, we have $e^{2 i \theta_{4}}=|f(0)|^{2}=1$. Thus,

$$
\overline{f(\bar{z})}=\frac{1}{f(z)} .
$$

To get a contradiction, we claim $f(z)$ has no poles on the line $\frac{\omega_{1}}{2}+i t$, for $t \in \mathbb{R}$. The claim can be proved as follows. By (2.33) and (2.37), for any $t \in \mathbb{R}$, we have

$$
f(-i t)=\frac{1}{f(i t)}=\frac{1}{f(i t)},
$$

which implies $f(i t)$ is a real for all $t \in \mathbb{R}$. Then (2.28) yields $e^{2 i \theta_{2}}= \pm 1$.

If $e^{2 i \theta_{2}}=1$, then $f(0)=f\left(\omega_{2}\right)$. Thus, $f$ has a pole on $\left(0, \omega_{2}\right)$. Otherwise, there exists a $i t_{0} \in\left(0, \omega_{2}\right)$ such that $f^{\prime}\left(i t_{0}\right)=0$, which yields a contradiction that $f^{\prime}$ can have zero only at $n \omega_{1}+m \omega_{2}$. Now suppose $f$ has a pole at $i t_{0}$. Then $i t_{0}$ is a zero of $\frac{f(z)}{f^{\prime}(z)}$. By the argument above, $i t_{0} \neq \frac{\omega_{2}}{2}$, and $-i t_{0}$ is also a zero of $\frac{f}{f^{\prime}}$. Thus, $i t_{0}$ and $-i t_{0}$ are the only zeros of $\frac{f(z)}{f^{\prime}(z)}$ on the torus $\left[0, \omega_{1}\right] \times\left[0, \omega_{2}\right]$. Therefore, $\frac{f}{f^{\prime}}$ has no zeros on $\frac{\omega_{1}}{2}+i t, t \in \mathbb{R}$. If $e^{2 i \theta_{2}}=-1$, then $f\left(\omega_{2}\right)=-f(0)$. If $f$ has a pole on $\left(0, \omega_{2}\right)$, then the argument above show $\frac{f(z)}{f^{\prime}(z)}$ has all zeros on $\left[0, \omega_{2}\right]$, and the claim is proved. If $f$ does not have a pole on $\left(0, \omega_{2}\right)$, then $f$ has a zero on $\left(0, \omega_{2}\right)$, say at $i t_{0}$. Since $i t_{0} \neq \frac{\omega_{2}}{2}$, we have $\omega_{2}-i t_{0} \neq i t_{0}$ is another zero of $\frac{f}{f^{\prime}}$. Thus, $\frac{f(z)}{f^{\prime}(z)}$ has no zeros on $\frac{\omega_{1}}{2}+i t, t \in \mathbb{R}$. The claim is proved.

Together with (2.33), we have

$$
\begin{aligned}
f\left(\frac{\omega_{1}}{2}+i t\right) & =\frac{1}{f\left(-\frac{\omega_{1}}{2}-i t\right)} \\
& =\overline{f\left(-\frac{\omega_{1}}{2}+i t\right)} \\
& =e^{2 i \theta_{1}} \overline{f\left(\frac{\omega_{1}}{2}+i t\right)},
\end{aligned}
$$

that is,

$$
\overline{f\left(\frac{\omega_{1}}{2}+i t\right) e^{-i \theta_{1}}}=e^{-i \theta_{1}} f\left(\frac{\omega_{1}}{2}+i t\right),
$$

which implies $g(t):=f\left(\frac{\omega_{1}}{2}+i t\right) e^{-i \theta_{1}}$ is a real-valued function for $t \in \mathbb{R}$. Since $g\left(2 \omega_{2}\right)=g(0)$ and $f(z)$ has no poles on the line $\frac{\omega_{1}}{2}+i t$, there exists $i t_{0} \in\left[0, \omega_{2}\right]$ such that

$$
f^{\prime}\left(\frac{\omega_{1}}{2}+i t_{0}\right)=0
$$


which yields a contradiction to the fact that $f^{\prime}$ can have zeros only at $n \omega_{1}+m \omega_{2}$.

For the second case, we have

$$
q_{2}=0,\left|p_{2}\right|=1 \text { and } A_{3}= \pm 1 .
$$

In this case, we have

$$
\overline{f(\bar{z})}=e^{2 i \theta_{5}} f(z)
$$

Since $f(0)=1$, it yields

$$
\overline{f(\bar{z})}=f(z)
$$

In particular, we have

$$
f(t) \in \mathbb{R} \text { for all } t \in \mathbb{R} .
$$

Hence by (2.28), $e^{2 i \theta_{1}}= \pm 1$, and

$$
f\left(z+2 \omega_{1}\right)=f(z) .
$$

By using the same argument as above, we can prove that $f$ has no poles on the line $\frac{\omega_{2}}{2}+t, t \in \mathbb{R}$. Now consider

$$
f\left(\frac{\omega_{2}}{2}+t\right)=\overline{f\left(-\frac{\omega_{2}}{2}+t\right)}=\overline{f\left(\frac{\omega_{2}}{2}+t\right) e^{-2 i \theta_{2}}}=\overline{f\left(\frac{\omega_{2}}{2}+t\right)} e^{2 i \theta_{2}}
$$

Thus,

$$
\overline{f\left(\frac{\omega_{2}}{2}+t\right) e^{-i \theta_{2}}}=\overline{f\left(\frac{\omega_{2}}{2}+t\right)} e^{i \theta_{2}}=f\left(\frac{\omega_{2}}{2}+t\right) e^{-i \theta_{2}},
$$

that is

$$
f\left(\frac{\omega_{2}}{2}+t\right) e^{-i \theta_{2}} \in \mathbb{R} \text { for all } t \in \mathbb{R} .
$$

Since $f\left(\frac{\omega_{2}}{2}\right)=f\left(\frac{\omega_{2}}{2}+2 \omega_{1}\right)$, there exists $t_{0} \in\left(0,2 \omega_{1}\right)$ such that

$$
f^{\prime}\left(\frac{\omega_{2}}{2}+t_{0}\right)=0
$$

which yields a contradiction. It completes the proof for case (ii).

CASE (iii). In this case, $S_{1}= \pm i d$. Hence $f$ satisfies

$$
f\left(z+\omega_{1}\right)=f(z) \text { for } z \in \mathbb{C} .
$$

By Lemma 2.4, there exists a $H=\left(\begin{array}{cc}p & -\bar{q} \\ q & \bar{p}\end{array}\right)$ such that

$$
H S_{2} H^{-1}=\left(\begin{array}{cc}
e^{i \theta} & 0 \\
0 & e^{-i \theta}
\end{array}\right) \text { for some } \theta \in \mathbb{R} .
$$

Replace $f$ by $H f$, still denote it by $f$. It is obvious that (2.42) still holds. Furthermore, $f$ satisfies

$$
f\left(z+\omega_{2}\right)=e^{2 i \theta} f(z)
$$

Therefore, case (iii) is reduced to case (ii), and the proof of Theorem 1.1 is completed. 


\section{3. - A refined estimate}

In this section, we consider a sequence of solution $v_{j}$ of

$$
\Delta v_{j}+\rho\left(\frac{h(x) e^{v_{j}}}{\int h(x) e^{v_{j}}}-1\right)=0 \text { in } \Omega,
$$

where $h(x)=e^{-8 \pi G(x)}$ and $\lim _{j \rightarrow+\infty} \rho_{j}=8 \pi$. Set $u_{j}=v_{j}-\log \left(\int h(x) e^{v_{j}}\right)$. Then $u_{j}$ satisfies

$$
\Delta u_{j}+\rho_{j}\left(h(x) e^{u_{j}}-1\right)=0 \text { in } \Omega .
$$

In this section, we always assume

$$
\lambda_{j}=\max _{\Omega} u_{j}=u_{j}\left(p_{j}\right) \rightarrow+\infty
$$

as $j \rightarrow+\infty$ and $\lim _{j \rightarrow+\infty} p_{j}=p_{0}$. Then it is well-known that $p_{0}$ is a critical point of $h$. By Lemma 2.1, we have $p_{0}=\omega_{k} / 2$ for some $k=1,2,3$, where $w_{3}=\frac{1}{2}\left(\omega_{1}+\omega_{2}\right)$. The main purpose of this section is to prove

THEOREM 3.1. Let $v_{j}$ be a sequence of blow-up solutions of (3.1) and $\omega_{k} / 2$ is the only blow-up point of $v_{j}$ for some $k \in\{1,2,3\}$. Then there exists a constant $c_{k}$ such that

$$
\rho_{j}-8 \pi=\left(c_{k}+o(1)\right) e^{-\lambda_{j}} .
$$

Furthermore both $c_{1}$ and $c_{2}$ are positive and $c_{3}$ is negative.

To prove Theorem 3.1, we should recall some estimates from [11]. Let $u_{j}, \lambda_{j}$ and $p_{j}$ be defined in (3.2) and (3.3). Then Li [20] proved that there exist constants $c$ and $\delta_{0}$ such that

$$
\left|u_{j}(x)-\lambda_{j}+2 \log \left(1+\frac{\rho_{j} h\left(p_{j}\right)}{8} e^{\lambda_{j}}\left|x-p_{j}\right|^{2}\right)\right| \leq c
$$

for $\left|x-p_{j}\right| \leq \delta_{0}$. Let $\tilde{G}$ be regular part of the Green function $G(x, p)$,

$$
\tilde{G}(x, p)=G(x, p)+\frac{1}{2 \pi} \log |x-p|
$$

and

$$
\varphi(p)=\tilde{G}(p, p) .
$$

Since $\Omega$ has the flat metric, $\varphi(p)$ is a constant function in $\Omega$. To understand more precisely behavior of $u_{j}$ in ball $B\left(p_{0}, \delta_{0}\right)$, we let

$$
\eta_{j}(x)=u_{j}(x)-U_{j}(x)-\rho_{j}\left(\tilde{G}\left(x, p_{j}\right)-\varphi\left(p_{j}\right)\right),
$$


where

$$
U_{j}(x)=\lambda_{j}-2 \log \left(1+\frac{\rho_{j} h\left(p_{j}\right) e^{\lambda_{j}}}{8}\left|x-q_{j}\right|^{2}\right)
$$

and

$$
\nabla U_{j}\left(p_{j}\right)=\nabla \log h\left(p_{j}\right)
$$

This shifting of center $q_{j}$ from $p_{j}$ is very important for our refined estimates, because it can give us the following sharp estimate.

$$
\left|\eta_{j}(x)\right| \leq O\left(\lambda_{j} e^{-\lambda_{j}}\right) \text { for }\left|x-p_{0}\right| \leq \delta_{0} .
$$

Lemma 3.1. Let $\eta_{j}(x)$ be defined as in (3.7). Then $\eta_{j}$ satisfies the following equation

$$
\Delta \eta_{j}(x)+\rho_{j} h\left(p_{j}\right) e^{U_{j}(x)} H_{j}\left(x, \eta_{j}\right)=0 \text { for } x \in \Omega,
$$

where $H_{j}(x, t)$ is defined by

$$
H_{j}(x, t)=\frac{h(x)}{h\left(p_{j}\right)} e^{t+\rho_{j}\left(\tilde{G}\left(x, p_{j}\right)-\varphi\left(p_{j}\right)\right)}-1 .
$$

ProOF. (3.11) can be proved by a straightforward computation. Note $\Delta \tilde{G}\left(x, p_{j}\right)=1$ for $x \in \Omega$. Thus, we have

$$
\begin{aligned}
\Delta \eta_{j}(x) & =\rho_{j} h\left(p_{j}\right) e^{U_{j}(x)}-\rho_{j} h(x) e^{u_{j}} \\
& =-\rho_{j} h\left(p_{j}\right) e^{U_{j}(x)}\left(\frac{h(x)}{h\left(p_{j}\right)} e^{u_{j}-U_{j}}-1\right) \\
& =-\rho_{j} h\left(p_{j}\right) e^{U_{j}(x)}\left(\frac{h(x)}{h\left(p_{j}\right)} e^{\eta_{j}(x)+\rho_{j}\left(\tilde{G}\left(x, p_{j}\right)-\varphi\left(p_{j}\right)\right)}-1\right)
\end{aligned}
$$

where the last identity follows from (3.7).

Lemma 3.2. Let $v_{j}$ and $\rho_{j}$ be a pair of solutions of (3.1). Then

$$
\rho_{j}-8 \pi=\frac{8}{\pi h\left(p_{0}\right)}\left(\int_{\Omega} \frac{H(x, 0)}{\left|x-p_{0}\right|^{4}} d x-\int_{\Omega^{c}} \frac{d x}{\left|x-p_{0}\right|^{4}}+o(1)\right) e^{-\lambda_{j}}
$$

where $H(x, 0)$ is defined by

$$
H(x, 0)=\frac{h(x)}{h\left(p_{0}\right)} e^{8 \pi\left(\tilde{G}\left(x, p_{0}\right)-\varphi\left(p_{0}\right)\right)}-1
$$

and $\Omega^{c}=\mathbb{R}^{2} \backslash \Omega$. 
ProOF. We first recall

$$
\rho_{j} h(x) e^{u_{j}(x)}=\rho_{j} h\left(p_{j}\right) e^{U_{j}}+\rho_{j} h\left(p_{j}\right) e^{U_{j}} H_{j}\left(x, \eta_{j}(x)\right) .
$$

Then

$$
\begin{aligned}
\rho_{j} & =\int_{\Omega} \rho_{j} h e^{u_{j}(x)} d x \\
& =\int_{\Omega} \rho_{j} h\left(p_{j}\right) e^{U_{j}} d x+\int_{\Omega} \rho_{j} h\left(p_{j}\right) e^{U_{j}} H_{j}\left(x, \eta_{j}(x)\right) d x \\
& =8 \pi-\int_{\Omega^{c}} \rho_{j} h\left(p_{j}\right) e^{U_{j}} d x+\int_{\Omega} \rho_{j} h\left(p_{j}\right) e^{U_{j}} H_{j}\left(x, \eta_{j}(x)\right) d x
\end{aligned}
$$

By the explicit expression of $U_{j}$, we have

$$
\int_{\Omega^{c}} \rho_{j} h\left(p_{j}\right) e^{U_{j}(x)} d x=\left(\frac{8}{\pi h\left(p_{0}\right)} \int_{\Omega^{c}} \frac{d y}{\left|y-p_{0}\right|^{4}}+o(1)\right) e^{-\lambda_{j}}
$$

For the second integral, we apply a useful trick from [11]. Set

$$
\psi_{j}(x)=\frac{1-a_{j}\left|x-q_{j}\right|^{2}}{1+a_{j}\left|x-q_{j}\right|^{2}}
$$

where

$$
a_{j}=\frac{\rho_{j} h\left(p_{j}\right)}{8} e^{\lambda_{j}}
$$

We use $\psi_{j}(x)$ as a comparison function because it satisfies

$$
\Delta \psi_{j}+\rho_{j} h\left(p_{j}\right) e^{U_{j}(x)} \psi_{j}=0 \text { in } \mathbb{R}^{2} .
$$

Thus, together with (3.11),

$$
\int_{\partial \Omega}\left(\psi_{j} \frac{\partial \eta_{j}}{\partial v}-\eta_{j} \frac{\partial \psi_{j}}{\partial v}\right) d \sigma=-\int_{\Omega} \rho_{j} h\left(p_{j}\right) e^{U_{j}(x)}\left(H_{j}\left(x, \eta_{j}(x)\right)-\eta_{j}\right) \psi_{j} d x .
$$

By noting

$$
\psi_{j}=-1+\frac{2}{1+a_{j}\left|x-q_{j}\right|^{2}}
$$

we have

$$
\begin{aligned}
& \int_{\Omega} \rho_{j} h\left(p_{j}\right) e^{U_{j}} H_{j}(x, 0)\left(-\psi_{j}\right) d x \\
& =\int_{\Omega} \rho_{j} h\left(p_{j}\right) e^{U_{j}} H_{j}(x, 0) d x-2 \int_{\Omega} \frac{\rho_{j} h\left(p_{j}\right) e^{U_{j}}}{1+a_{j}\left|x-p_{0}\right|^{2}} H_{j}(x, 0) d x .
\end{aligned}
$$


By (3.13), we have

$$
\begin{aligned}
h\left(p_{j}\right) H_{j}(x, 0)= & \exp \left\{\log (h)+\rho\left[\tilde{G}\left(x, p_{j}\right)-\varphi\left(p_{j}\right)\right]\right\}-h\left(p_{j}\right) \\
= & h\left(p_{j}\right)\left\{\sum_{k=1}^{2} b_{k}\left(x_{k}-p_{j, k}\right)+\frac{1}{2} \sum_{i, k=1}^{2} B_{i k}\left(x_{i}-p_{j, i}\right)\left(x_{k}-p_{j, k}\right)\right. \\
& \left.+\frac{1}{2}\left[\sum_{k=1}^{2} b_{k}\left(x_{k}-p_{j, k}\right)\right]^{2}\right\}+O\left(\left|x-p_{j}\right|^{3}\right),
\end{aligned}
$$

where $b=\left(b_{1}, b_{2}\right)=\nabla\left[\log (h)+\rho_{j} \tilde{G}\left(x, p_{j}\right)\right]$ at $x=p_{j},\left(B_{i k}\right)$ is the Hessian of $\log (h)+\rho_{j} \tilde{G}\left(x, p_{j}\right)$ at $x=p_{j}$ and $p_{j}=\left(p_{j, 1}, p_{j, 2}\right)$. Since

$$
\begin{gathered}
B_{11}+B_{22}=\Delta\left[\log (h)+\rho_{j} \tilde{G}\left(x, p_{j}\right)\right]=8 \pi-\rho_{j}, \\
|b| \leq c \quad \lambda_{j} e^{-\lambda_{j}} \quad \text { and } \quad\left|p_{j}-q_{j}\right| \leq c e^{-\lambda_{j}},
\end{gathered}
$$

we have for any $\delta>0$,

$$
\rho_{j} \int_{B\left(p_{j}, \delta\right)} h\left(p_{j}\right) e^{U_{j}(x)} H_{j}(x, 0) d x=e^{-\lambda_{j}}\left(o(1)+o_{\delta}(1)\right),
$$

where $o_{\delta}(1) \rightarrow 0$ as $\delta \downarrow 0$ and $o(1) \rightarrow 0$ as $j \rightarrow+\infty$. Thus,

$$
\rho_{j} \int_{\Omega} h\left(p_{j}\right) e^{U_{j}(x)} H_{j}(x, 0) d x=\frac{8}{\pi h\left(p_{0}\right)} e^{-\lambda_{j}}\left(\int_{\Omega} \frac{H(x, 0)}{\left|x-p_{0}\right|^{4}} d x+o(1)\right) .
$$

By using the scaling $x^{\prime}=e^{\frac{\lambda_{j}}{2}} x$, we can obtain

$$
\int_{\Omega} \frac{e^{U_{j}(x)}}{1+a_{j}\left|x-p_{0}\right|^{2}} H_{j}(x, 0) d x=O\left(\lambda_{j} e^{-\frac{3}{2} \lambda_{j}}\right) .
$$

By (3.11), $H_{j}\left(x, \eta_{j}(x)\right)-\eta_{j}=H_{j}(x, 0)+H_{j}(x, 0) \eta_{j}+O\left(\left|\eta_{j}\right|^{2}\right)$. Thus it is easy to see the integration of $H_{j}(x, 0) \eta_{j} \psi_{j}$ and $\left|\eta_{j}\right|^{2} \psi_{j}$ is bounded by $o(1) e^{-\lambda_{j}}$. (3.18) and (3.19) give

$$
\int_{\partial \Omega}\left(\psi_{j} \frac{\partial \eta_{j}}{\partial v}-\eta_{j} \frac{\partial \psi_{j}}{\partial v}\right) d \sigma=\frac{8}{\pi h\left(p_{0}\right)} e^{-\lambda_{j}}\left(\int_{\Omega} \frac{H(x, 0)}{\left|x-p_{0}\right|^{4}} d x+o(1)\right) .
$$

Since $\psi_{j}+1$ and $\nabla \psi_{j}=O\left(e^{-\lambda_{j}}\right)$, we have

$$
\begin{aligned}
& \int_{\Omega} \rho_{j} h\left(p_{j}\right) e^{U_{j}} H_{j}\left(x, \eta_{j}(x)\right) d x \\
& =-\int_{\partial \Omega} \frac{\partial \eta_{j}}{\partial v} d \sigma \\
& =\int_{\partial \Omega}\left(\psi_{j} \frac{\partial \eta_{j}}{\partial v}-\eta_{j} \frac{\partial \psi_{j}}{\partial v}\right) d \sigma+o\left(e^{-\lambda_{j}}\right) \\
& =\frac{8}{\pi h\left(p_{0}\right)} e^{-\lambda_{j}}\left(\int_{\Omega} \frac{H(x, 0)}{\left|x-p_{0}\right|^{4}} d x+o(1)\right) .
\end{aligned}
$$

Together with (3.14), this completes the proof of Lemma 3.2. 
Set

$$
l\left(p_{0}\right)=\int_{\Omega} \frac{h(x) e^{8 \pi\left(\tilde{G}\left(x, p_{0}\right)-\varphi\left(p_{0}\right)\right)}-h\left(p_{0}\right)}{\left|x-p_{0}\right|^{4}}-\int_{\Omega^{c}} \frac{h\left(p_{0}\right)}{\left|x-p_{0}\right|^{4}}
$$

where by Lemma 3.2, $l\left(p_{0}\right)=c_{k}$ if $p_{0}=\frac{\omega_{k}}{2}$. Clearly, the integral of (3.21) is interpreted as

$$
\begin{aligned}
& l\left(p_{0}\right) \\
& =\lim _{r \rightarrow 0}\left[\int_{\Omega \backslash B_{r}\left(p_{0}\right)} \frac{h(x) e^{8 \pi\left(\tilde{G}\left(x, p_{0}\right)-\varphi\left(p_{0}\right)\right)}}{\left|x-p_{0}\right|^{4}}-\int_{\mathbb{R}^{2} \backslash B_{r}\left(p_{0}\right)} \frac{h\left(p_{0}\right)}{\left|x-p_{0}\right|^{4}}\right] \\
& =\lim _{r \rightarrow 0} e^{-8 \pi \varphi\left(p_{0}\right)}\left[\int_{\Omega \backslash B_{r}\left(p_{0}\right)} e^{8 \pi\left(G\left(x, p_{0}\right)-G(x)\right)}-\int_{\mathbb{R}^{2} \backslash B_{r}\left(p_{0}\right)} \frac{e^{8 \pi\left(\varphi\left(p_{0}\right)-G\left(p_{0}\right)\right)}}{\left|x-p_{0}\right|^{4}}\right] .
\end{aligned}
$$

To compute $l\left(p_{0}\right)$, we should give a geometric interpretation of the integral. Note $4 \pi\left(G\left(x, p_{0}\right)-G(x)\right)$ is a doubly-periodic harmonic function in $\mathbb{R}^{2}$ and has a singularity $-2 \log \left|x-p_{0}\right|$ at $p_{0}$ and $2 \log |x|$ at 0 . Thus,

$$
4 \pi\left(G\left(x, p_{0}\right)-G(x)\right)=\log \left|\wp\left(x-p_{0}\right)-\wp\left(p_{0}\right)\right|+\text { a constant }
$$

where $\wp$ is the Weierstrass elliptic function. In other words,

$$
e^{8 \pi\left(G\left(x, p_{0}\right)-G(x)\right)}=e^{8 \pi\left(\varphi\left(p_{0}\right)-G\left(p_{0}\right)\right)}\left|\wp\left(z-p_{0}\right)-\wp\left(p_{0}\right)\right|^{2},
$$

where $z=x_{1}+i x_{2}$. Thus,

$$
l\left(p_{0}\right)=e^{-8 \pi G\left(p_{0}\right)} \lim _{r \rightarrow 0}\left[\int_{\Omega \backslash B_{r}\left(p_{0}\right)}\left|\wp\left(z-p_{0}\right)-\wp\left(p_{0}\right)\right|^{2}-\int_{\mathbb{R}^{2} \backslash B_{r}\left(p_{0}\right)} \frac{1}{\left|x-p_{0}\right|^{4}}\right] .
$$

Set $\sigma(z)$ to denote the antiderivative of $\wp\left(z-p_{0}\right)-\wp\left(p_{0}\right)$. Then

$$
l(p)=e^{-8 \pi G\left(p_{0}\right)} \lim _{r \rightarrow 0}\left[\int_{\Omega \backslash B_{r}\left(p_{0}\right)}\left|\sigma^{\prime}(z)\right|^{2}-\int_{\mathbb{R}^{2} \backslash B_{r}\left(p_{0}\right)} \frac{1}{\left|x-p_{0}\right|^{4}}\right] .
$$

Clearly, $\int_{\Omega \backslash B_{r}\left(p_{0}\right)}\left|\sigma^{\prime}(x)\right|^{2}$ can be interpreted as the area of the image of $\Omega \backslash B_{r}\left(p_{0}\right)$ under $\sigma(z)$, where $\sigma(z)$ has a singularity at $p_{0}$. Also, the second integral is the area of the image of $\mathbb{R}^{2} \backslash B_{r}\left(p_{0}\right)$ under the map $\frac{1}{z-p_{0}}$. Let $\Lambda$ be the closed curve which is the image of $\partial \Omega$ under $\sigma(z)$. By a straightforward computation, the image of $\partial B_{r}\left(p_{0}\right)$ under $\sigma(z)$ is contained in the annulus

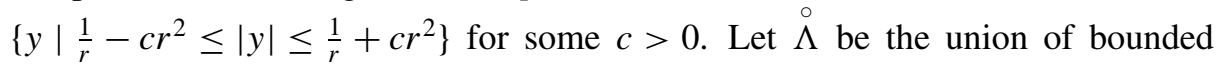
components of $\mathbb{R}^{2} \backslash \Lambda$. Thus,

$$
\begin{gathered}
l\left(p_{0}\right)=e^{-8 \pi G\left(p_{0}\right)} \text {, the area of the components of } \stackrel{\circ}{\Lambda} \text { which is covered } \\
\text { by } \sigma(z) \text { minus the area of } \stackrel{\circ}{\Lambda}\} .
\end{gathered}
$$


Note the component covered by $\sigma(z)$ has to be counted in the multiplicity of the covering by $\sigma(z)$. In the following, we will show that $\Lambda$ is actually a rectangle and the interior $\Lambda$ of $\Lambda$ could not be covered by $\sigma(z)$ when $p_{0}=\frac{\omega_{3}}{2}$, and each point of $\Lambda$ is covered by $\sigma(z)$ at least twice when $p_{0}=\frac{w_{1}}{2}$ of $\frac{\omega_{2}}{2}$. Therefore $l\left(p_{0}\right)>0$ if $p_{0}=\frac{\omega_{1}}{2}$ or $\frac{\omega_{2}}{2}$ and $l\left(p_{0}\right)<0$ if $p_{0}=\frac{\omega_{3}}{2}$. Clearly, Theorem 3.1 follows readily from our claim.

To prove our claim, without loss of generality, we may consider the fundamental cell to be the rectangle which has $p_{0}$ as its center. By the elementary theory of $\wp, \wp\left(z-p_{0}\right)$ is real-valued for $z \in \partial \Omega$. Furthermore, $\wp\left(z-p_{0}\right)-\wp\left(p_{0}\right) \geq 0$ for $z \in \partial \Omega$ and $p_{0}=\frac{\omega_{2}}{2}, \wp\left(z-p_{0}\right)-\wp\left(p_{0}\right) \leq 0$ for $z \in \partial \Omega$ and $p_{0}=\frac{\omega_{1}}{2}$, and $\wp\left(z-p_{0}\right)-\wp\left(p_{0}\right)<0$ for $z-p_{0}= \pm \frac{\omega_{1}}{2}+i t, t \in \mathbb{R}$ and $\wp\left(z-p_{0}\right)-\wp\left(p_{0}\right)>0 z-p_{0}= \pm \frac{\omega_{2}}{2}+t, t \in R$ if $p_{0}=\frac{\omega_{3}^{2}}{2}$. From this fact, it is easy to see that $\sigma$ maps $\partial \Omega 1-1$ and onto a rectangle $\Lambda$.

Now we consider the case $p_{0}=\frac{\omega_{3}}{2}$. In this case, the origin is at the corner of $\partial \Omega$. Since $\sigma(z)=\sigma(0)+\frac{\wp^{\prime \prime}\left(p_{0}\right)}{6} z^{3}+O\left(|z|^{4}\right)$ and $\wp^{\prime \prime}\left(p_{0}\right)$ is negative number, $\left\{z=r e^{i \theta} \mid 0 \leq \theta<\frac{\pi}{2}, 0 \leq r<r_{0}\right\}$ is mapped onto the outside of $\Lambda$ at the corner $\sigma(0)$. See Figure 1 below. We claim that $\sigma(\Omega)=\mathbb{R}^{2} \backslash \Lambda$. Note that for each $Q \in \mathbb{R}^{2} \backslash \Lambda$, there exists a unique point $q \in \Omega^{\circ}$, the interior of $\Omega$ such that $\sigma(Q)=q$. Now suppose $\sigma(\Omega) \supset \mathbb{R}^{2} \backslash \Lambda$. Then there exists a sequence of point $q_{n} \in \Lambda$, the interior of $\Lambda$, and $Q_{n} \in \Omega$ such that $q_{n}=\sigma\left(Q_{n}\right) \rightarrow \sigma(0)$. By Figure 1 below, $\left|Q_{n}\right| \geq r_{0}$. Let $Q$ be a limit point of $Q_{n}$. Then $\sigma(Q)=\sigma(0)$ and $Q \neq 0$. Thus $Q \notin \partial \Omega$ because $\sigma$ is $1-1$ on $\partial \Omega$. Then the image of a neighborhood of $Q$ also covers a neighborhood of $\sigma(0)$. In particular, any point in a neighborhood of $\sigma(0)$ outside of $\Lambda$ contains at least two preimages, which yields a contradiction. Thus, our claim is proved when $p_{0}=\frac{\omega_{3}}{2}$.

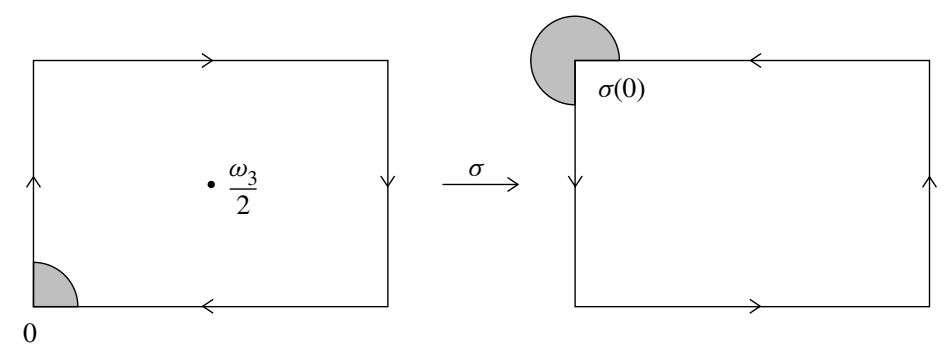

Fig. 1.

For the case $p_{0}=\frac{\omega_{1}}{2}$, we have 0 on the line of $\partial \Omega$, but not at the corner. Since $\sigma(z)=\sigma(0)+\frac{\wp\left(p_{0}\right)}{6} z^{3}+O\left(|z|^{4}\right)$ and $\wp^{\prime \prime}\left(p_{0}\right)>0$ is real, $\left\{z=r e^{i \theta} \mid 0 \leq\right.$ $r<r_{0}$ and $\left.-\frac{\pi}{2} \leq \theta \leq \frac{\pi}{2}\right\}$ is mapped into $\Lambda^{\circ}$ and covers each image twice. See Figure 2. 


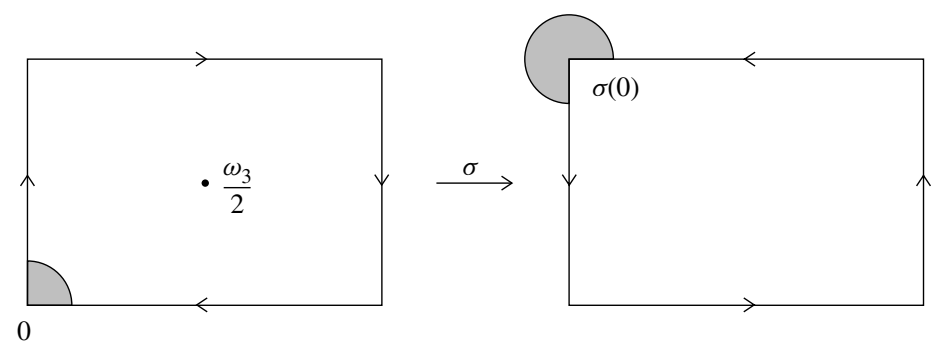

Fig. 2.

Clearly, the image of $\Omega$ under $\sigma$ must at least cover each point in $\stackrel{\circ}{\Lambda}$ twice. Therefore our claim is proved. The proof for the case $p_{0}=\frac{\omega_{2}}{2}$ is similar. Hence the proof of Theorem 3.1 is completed.

\section{4. - Counting the topological degree}

In this section, we want to count the topological degree for $\rho=8 \pi$ and $\rho \in(8 \pi, 16 \pi)$. In [12], the topological degree has been computed when the potential $h(x)$ is a positive function. For our case, $h(x)$ always satisfies (1.17) at vanishing points. It is clear that for a sequence of blow-up solution $v_{j}$ of (1.15) $p_{0}$ is the only blow-up point. If $h(x)$ vanishes at $p_{0}$, then $\rho_{j}$ must satisfy

$$
\lim _{j \rightarrow+\infty} \rho_{j}=8 \pi(1+\alpha) .
$$

where $2 \alpha$ is the vanishing order of $h$ at $p_{0}$. Therefore, if we are restricted to the situation $\rho<8 \pi(1+\alpha)$, then the blow-up can occur at some non-vanishing point of $h$. Thus, the results in [12] can be applied to our work here. For the sake of convenience, we quote some useful results from [12] in the followings:

(1) Let $M$ be a compact Riemann surface, $h(x)$ a positive $C^{2}$ function in $M \backslash\left\{p_{1}, \ldots, p_{m}\right\}$ and $\log (h(x))+4 \pi \varphi(x)$ a Morse function in $M \backslash\left\{p_{1}, \ldots, p_{m}\right\}$ where $\varphi(x)$ is the regular part of the Green function. Then at any critical point $p$ of $\log h+4 \pi \varphi(x)$ where $h(p)>0$, there exists only a sequence of blow-up solution $v_{j}$ of (1.15) with $\rho=\rho_{j}$ such that $\lim _{j \rightarrow+\infty} \rho_{j}=8 \pi$ and $p$ is the only blow-up point of $v_{j}$.

(2) The topological index for the solution $v_{j}$ can be counted as $(-1)^{\operatorname{ind}_{p} \log (h)+4 \pi \varphi}$ if $\rho_{j}<8 \pi$, and $-(-1)^{\operatorname{ind}_{p} \log (h)+4 \pi \varphi}$ if $\rho_{j}>8 \pi$. Here $\operatorname{ind}_{p} \log (h)$ is the Morse index of $\log (h)$ at the critical point $p$.

(3) Suppose $h(x)$ satisfies

$\Delta \log h(p)+8 \pi-2 K(p) \neq 0$, and $p$ is a critical point of $\log h(x)+4 \pi \varphi(x)$. 
Then

$$
\rho_{j}-8 \pi=\frac{2}{h(p)}(\Delta \log h(p)+8 \pi-2 K(x)+o(1)) \lambda_{j} e^{-\lambda_{j}},
$$

where $K(x)$ is the Gaussian curvature

$$
\lambda_{j}=\max _{\Omega} v_{j}-\log \left(\int h(x) e^{v_{j}}\right) .
$$

For the proof of (1)-(3), we refer to [11] and [12].

Now we are in the position to complete the proof of Theorem 1.2 and Theorem 1.3.

Proof of Theorem 1.2. By Lemma 2.1, the potential $h(x)=e^{-8 \pi G(x)}$ is a Morse function and has only three critical points $\frac{\omega_{1}}{2}, \frac{\omega_{2}}{2}$ and $\frac{\omega_{3}}{2}$. Furthermore, ind $_{p} h=1$ if $p=\frac{\omega_{1}}{2}$, or $\frac{\omega_{2}}{2}$ and $\operatorname{ind}_{p} h=0$ if $p=\frac{\omega_{3}}{2}$. By Theorem 3.1, we have proved the uniform boundedness of all solutions of (1.15) when $\rho=8 \pi$. Together (1) and (2) prove that there only exists a sequence of blow-up solution $v_{j}$ such that $\rho_{j}<8 \pi$ and $\lim _{j \rightarrow+\infty} \rho_{j}=8 \pi$. This is the case with $p=\frac{\omega_{3}}{2}$. Thus, the index of $v_{j}$ is 1 . Let $d_{\rho}$ denote the topological degree of (1.15). It is known that $d_{\rho}=1$ if $\rho<8 \pi$. Thus

$$
d_{8 \pi}=d_{\rho}-\text { the index of } v_{j}=1-1=0 .
$$

This completes the proof of Theorem 1.2.

Theorem 3.1 actually allows us to calculate the degree of (1.15) when $h(x)$ vanishes only at one point. By Theorem 3.1, we know there are only two sequences of blow-up solutions of (1.15) with the blow-up point $p=\frac{\omega_{1}}{2}$ or $p=\frac{\omega_{2}}{2}$. Since the Hessian of $h(x)$ has one negative eigenvalue and $\rho_{j}>8 \pi$, the topological index of each blow-up solution is equal to 1 . Thus for $\rho<$ $(8 \pi, 16 \pi)$,

$$
d_{\rho}=d_{8 \pi}+2=2 .
$$

For the general case, we follow a similar way of calculation.

Proof of Theorem 1.3. Let $M$ be a compact Riemann surface and $g$ be the genus. Without loss of generality, we assume $M$ has constant Gauss curvature. Hence the regular part $\varphi(x)$ is a constant on $M$. If the genus of $M$ is greater than 1, we first choose a positive Morse function $h(x)$ in $M \backslash\left\{p_{1}, \ldots, p_{m}\right\}$ and $h\left(p_{j}\right)=0$ for $j=1,2, \ldots, m$, and then replace it by $h^{\varepsilon}(x)$ for some $\varepsilon>0$ such that

$$
\Delta h^{\varepsilon}(x)+8 \pi-2 K(p)>0 \text { for } \quad x \in M \backslash\left\{p_{1}, \ldots, p_{m}\right\} .
$$


For convenience, we denote $h^{\varepsilon}(x)$ by $h_{0}(x)$. If $M$ is the sphere, the Gaussian curvature $K=4 \pi$. Thus, we choose a positive Morse function $h_{0}$ or $M \backslash\left\{p_{1}, \ldots, p_{m}\right\}$ with $h_{0}\left(p_{j}\right)=0$ such that

$$
\Delta \log h_{0}(x) \neq 0 \text { for any critical point } x \neq p_{j} \text { of } h .
$$

Let

$$
h_{0}(x)=\left|x-p_{j}\right|^{2 \alpha_{j}} h_{j}(x) \quad j=1,2, \ldots, m,
$$

where $\alpha_{0}=\min \left(\alpha_{1}, \ldots, \alpha_{m}\right)>0$. We let $8 \pi<\rho<\min \left(16 \pi, 8 \pi\left(1+\alpha_{0}\right)\right)$. It is known that $d_{\rho}$ is independent of $\rho$. For any critical point $q_{l} \notin\left\{p_{1}, \ldots, p_{m}\right\}$ of $h(x)$, there exists a blow-up solution $v_{j}$ (3.15) with $\rho=\rho_{j}$ such that $q_{l}$ is the blow-up point. Furthermore, $\rho_{j} \neq 8 \pi$ due to (4.2) and (4.3). Hence, $d_{8 \pi}$ is well-defined and $d_{8 \pi}=d_{\rho}=1$ for any $\rho \in(0,8 \pi)$.

If $M \neq S^{2}$, then by (2) above, $v_{j}$ has the index $-(-1)^{i n d_{q_{l}} h}$. Thus, for $8 \pi<\rho<\min \left(16 \pi,\left(1+\alpha_{0}\right)\right)$,

$$
d_{\rho}=d_{8 \pi}-\sum_{l}(-1)^{i n q_{l} h_{0}},
$$

where $q_{l}, l=1, \ldots, N$ are the critical points of $h_{0}$. By the Morse Theorem,

$$
2-2 g-m=\chi\left(M \backslash\left\{p_{1}, \ldots, p_{m}\right)\right)=\sum_{l}(-1)^{i n d_{q_{l}} h_{0}} .
$$

Thus,

$$
d_{\rho}=m+2 g-1
$$

If $M=S^{2}$, then

$$
1=d_{\rho^{\prime}}=d_{8 \pi}+\sum_{\Gamma^{-}}(-1)^{\operatorname{ind}_{q} h_{0}}
$$

and

$$
d_{\rho}=d_{8 \pi}-\sum_{\Gamma^{+}}(-1)^{\operatorname{ind}_{q} h_{0}},
$$

where $\rho^{\prime}<8 \pi, 8 \pi<\rho<\min \left(16 \pi, 8 \pi\left(1+\alpha_{0}\right)\right)$ and $\Gamma^{ \pm}=\left\{x \in M \backslash\left\{p_{1}, \ldots, p_{l}\right\} \mid\right.$ $\Delta h_{0}(x)>0$ (or $\left.<0\right)$ and $x$ is a critical point of $\left.h_{0}\right\}$. Thus

$$
d_{\rho}=1-\sum_{\Gamma^{-} \cup \Gamma^{+}}(-1)^{\operatorname{ind}_{q} h_{0}}=1-\chi\left(M \backslash\left\{p_{1}, \ldots, p_{m}\right\}\right)=m-1 .
$$

It is easy to see that for some $h(x)$ which vanishes exactly at $p_{1}, \ldots, p_{m}$ we can deform $h_{t}(x)$ by $h_{t}(x)=h(t)^{t} h_{0}(t)^{1-t}$. And it is easy to see for some $16 \pi>\rho_{1}>8 \pi$, there is a constant $c>0$ such that $\|v(x)\|_{L^{\infty}} \leq c$ for any solution of (1.15) with $h=h_{t}$ and $8 \pi<\rho<\rho_{1}$. Thus, the degree $d_{\rho}$ is 
invariant for $0 \leq t \leq 1$. Since the vanishing order of $h \geq 1, d_{\rho}$ is the same for $\rho \in(8 \pi, 16 \pi)$. Thus, $d_{\rho}=m+2 g-1$ is proved.

In a similar way, we can compute the degree for the Dirichlet problem. As before, we let $G(x, y)$ be the Green function with singularity at $y$ and set the regular part $\varphi$ to be

$$
\varphi(x)=G(x, y)+\frac{1}{2 \pi} \log |x-y| \quad \text { at } \quad x=y .
$$

It is known that

$$
\varphi(x) \rightarrow-\infty \quad \text { as } \quad x \rightarrow \partial \Omega .
$$

Proof of Theorem 1.4. Let $h(x)=\exp -4 \pi\left(\sum_{j=1}^{m} n_{j} G\left(x, p_{j}\right)\right)$. Then we want to compute the topological degree of the following equation

$$
\left\{\begin{array}{l}
\Delta u+\rho \frac{h(x) e^{u(x)}}{\int_{\Omega} h(x) e^{u} d x}=0 \text { in } \Omega, \\
u=0 \text { on } \partial \Omega
\end{array}\right.
$$

As before, a function $h_{0}(x) \in C^{2}\left(\Omega \backslash\left\{p_{1}, \ldots, p_{m}\right\}\right.$ is chosen so that $\log h_{0}(x)+$ $4 \pi \varphi(x)$ is a Morse function, $h_{0}\left(p_{j}\right)=0$ and (1.17) holds for each $p_{j}$. We also require that $h_{0}$ satisfies

$$
\Delta \log h_{0}(x) \neq 0 \text { at any critical point of } \log h_{0}(x)+4 \pi \varphi(x) .
$$

Thus, by (4.9) the uniform boundedness of solutions of (4.8) with $h=h_{0}$ and $\rho=8 \pi$ is obtained, and then $d_{8 \pi}$ is well-defined. In a similar way, (4.7) and (4.8) are treated. We have, for $8 \pi<\rho<8 \pi\left(1+\alpha_{0}\right)$,

$$
d_{\rho}=1-\sum_{\Gamma}(-1)^{\operatorname{ind}_{q}\left(\log h_{0}+4 \pi \varphi\right)}=1-\chi\left(\Omega \backslash\left\{p_{1}, \ldots, p_{m}\right\}\right)=g+m,
$$

where $\Gamma=\left\{x \mid \nabla\left(\log h_{0}+4 \pi \varphi\right)(x)=0\right\}$ and $g$ is the number of holes inside $\Omega$. Here we note that $\log h_{0}+4 \pi \varphi(x) \rightarrow-\infty$ as $x \rightarrow \partial \Omega$ and the Morse theorem can be applied as well. Then we can deform $h_{0}$ to our original $h(x)$ and obtain the degree $d_{\rho}=g+m$ for our equation and $\rho \in(8 \pi, 16 \pi)$. Since $m \geq 1$, we have $d_{\rho} \neq 0$. Hence the existence follows readily.

\section{REFERENCES}

[1] L. Ahlfors, “Complex analysis", 2nd edition, McGraw-Hill Book Co., New York, 1966.

[2] D. BARTOLUCCI - G. TARANTEllo, Liouville type equations with singular data and their application to periodic multivortices for the electroweak theory, Comm. Math. Phys. 229 (2002), 3-47. 
[3] H. BREZIS - F. MERLE, Uniform estimates and blow-up behavior for solutions of $-\Delta u=$ $V(x) e^{u}$ in two dimensions, Comm. Partial Differential Equation 16 (1991), 1223-1253.

[4] R. L. BRYANT, Surfaces of mean curvature one in hyperbolic space, Astérisque 154-155 (1987), 321-347.

[5] L. Caffarelli - Y. Yang, Vortex condensation in the Chern-Simons Higgs model: an existence theorem, Comm. Math. Phys. 168 (1995), 321-336.

[6] E. Caglioti - P. L. Lions - C. Marchioro - M. Pulvirenti, A special class of stationary flows for two-dimensional Euler equations: A statistical mechanics description, Comm. Math. Phys. 143 (1992), 501-525.

[7] H. ChAN - C. C. Fu - C. S. Lin, Non-topological multivortex solutions to the self-dual Chern-Simons-Higgs equations, Comm. Math. Phys. 231 (2002), 189-221.

[8] S. Chanillo - M. Kiessling, Rotational symmetry of solutions of some nonlinear problems in statistical mechanics and in geometry, Comm. Math. Phys. 160 (1994), 217-238.

[9] S. Y. Chang - P. Yang, Prescribing Gaussian curvature on $S^{2}$, Acta Math. 159 (1987), 215-259.

[10] C. C. Chen - C. S. Lin, On the symmetry of blowup solutions to a mean field equation, Ann. Inst. H. Poincaré Anal. Non Linéaire 18 (2001), 271-296.

[11] C. C. CHen - C. S. Lin, Sharp estimates for solutions of multi-bubbles in compact Riemann surfaces, Comm. Pure Appl. Math. 4 (2002), 728-771.

[12] C. C. Chen and C. S. Lin, Topological Degree for a Mean Field Equation on Riemann Surfaces, Comm. Pure Appl. Math. 56 (2003), 1667-1707.

[13] K. S. Chou - Tom Y. H. Wan, Asymptotic radial symmetry for solutions of $\Delta u+e^{u}=0$ in a punctured disc, Pacific J. Math. 163 (1994), 269-276.

[14] W. Ding - J. Jost - J. Li - G. Wang, Multiplicity results of two-vortex Chern-SimonsHiggs model on the two-sphere, Comm. Math. Helv. 74 (1999), 118-142.

[15] W. Ding - J. Jost - J. Li - G. WANG, The differential equation of $\Delta u=8 \pi-8 \pi$ he $u$ a compact Riemann surface, Asian J. Math., 1 (1997), 230-248.

[16] W. Ding - J. Jost - J. Li - X. Peng - G. Wang, Self duality equations for GinzburgLandau and Seiberg-Witten type functional with 6th order potenliatls, Comm. Math. Phys. 217 (2001), 383-407.

[17] G. Dunne, "Self-dual Chern-Simons Theories", Lecture Notes in Physics m36, SpringerVerlag, Berlin, 1995.

[18] J. Hong - Y. KIm - P. Y. PaC, Multivortex solutions of the Abelian Chern Simons theory, Phys. Rev. Letter 64 (1990), 2230-2233.

[19] R. JACKiw - E. J. Weinberg, Selfdual Chern Simons vortices, Phys. Rev. Lett. 64 (1990), 2234-2237.

[20] Y. Y. LI, Harnack type inequality: the method of moving planes, Comm. Math. Phys. 200 (1999), 421-444.

[21] C. S. Lin, Topological degree for mean field equations on $S^{2}$, Duke Math. J. 104 (2000), 501-536.

[22] C. S. Lin, Uniqueness of solutions to the mean field equations for the spherical Onsager vortex, Arch. Ration. Mech. Anal. 153 (2000), 153-176.

[23] M. NolASCO, Non-topological N-vortex condensates for the self-dual chern-Simons theory, Comm. Pure Appl. Math. 56 (2003), 1752-1780. 
[24] M. Nolasco - G. Tarantello, Double vortex condensates in the Chern-Simons-Higgs theory, Calc. Var. Partial Differential Equations 9 (1999), 31-94.

[25] M. Nolasco - G. Tarantello, On a sharp Sobolev-type inequality on two-dimensional compact manifolds, Arch. Ration. Mech. Anal. 145 (1998), 161-195.

[26] J. Prajapat - G. Tarantello, On a class of elliptic problems in $\mathbb{R}^{2}$ : symmetry and uniqueness results, Proc. Roy. Soc. Edinburgh Sect. A 131 (2001), 967-985.

[27] SpRUCK - Y. YANG, Topological solutions in the self-dual Chern-Simons theory: existence and approximation, Ann. Inst. H. Poincaré Anal. Non Linéaire 12 (1995), 75-97.

[28] TAUBES, Arbitrary N-vortex solutions to the first order Ginzburg-Landau equations, Comm. Math. Phys. 72 (1980), 277-292.

Department of Mathematics Taiwan University

Taipei, Taiwan

chchchen@math.ntu.edu.tw

Department of Mathematics Chung-Cheng University Minghsiung, Chia-Yi 621

Taiwan

cslin@math.ccu.edu.tw

Max-Planck-Institute for

Mathematics in the Sciences

Inselstr. 22-26

04103 Leipzig, Germany

gwang@mis.mpg.de 